\title{
Recursive analytical performance evaluation of broadcast protocols with silencing: application to VANETs
}

\author{
Stefano Busanelli, Gianluigi Ferrari and Roberto Gruppini
}

\begin{abstract}
In this article, we present a novel theoretical framework suitable for analytical performance evaluation of a family of multihop broadcast protocols. The framework allows to derive several average performance metrics, including reliability, latency, and efficiency, and it is targeted to Vehicular Ad-hoc NETworks (VANETs) applications based on an underlying IEEE 802.11 protocol. It builds on the assumption that the positions of the nodes of a VANET can be statistically modeled as Poisson points. However, the proposed approach holds for any spatial vehicle distribution with constant average distance between consecutive vehicles. In this work, the proposed analytical framework is applied to the class of probabilistic broadcast multihop protocols with silencing, but can be generalized to nonprobabilistic protocols as well. More specifically, this work considers a few broadcast protocols with silencing, differing for the probability assignment function. The validity of the proposed analytical approach is assessed by means of numerical simulations in a highway-like scenario.
\end{abstract}

Keywords: poisson point process, VANET, broadcast protocol, performance analysis, IEEE 802.11, ns-2, highway, VanetMobiSim

\section{Introduction}

Nowadays, most of the vehicles available on the market are provided by sensorial, cognitive, and communication skills. In particular, leveraging on inter-vehicular communications-a set of technologies that gives networking capabilities to the vehicles-vehicles can create decentralized and self-organized vehicular networks, commonly denoted as vehicular Ad-hoc NETworks (VANETs), involving either vehicles and/or fixed network nodes (e. g., road side units).

Vehicular Ad-hoc NETworks present a few unique characteristics: (i) the availability of virtually unlimited energetic and computational resources (in each vehicle); (ii) very dynamic network topologies, due to the high average speed of the vehicles; (iii) nodes' movements constrained by the underlying road topology; (iv) the need for broadcast communication protocols, used as truly information-bearing protocols (especially in multihop communication scenarios) and not only as auxiliary

\footnotetext{
* Correspondence: stefano.busanelli@unipr.it

* Correspondence: stefano.busanelli@unipr.it
Department of Information Engineering, University of Parma, Viale G.P. Usberti 181/A, 43124 Parma, Italy
}

supporting tools. For instance, a multihop broadcast protocol fulfills well the requirements of applications such as the diffusion of safety-related messages (e.g., warning alerts) or public interest information (e.g., road interruptions).

Reducing the number of redundant packets, while still ensuring good coverage and low latency, is one of the main objectives in multi-hop broadcasting. In fact, a too large number of transmissions acts unavoidably leads to unsustainable levels of latency, retransmissions, and collisions: the overall phenomenon is typically referred to as broadcast storm problem [1] and it mainly affects dense networks. The problem of minimizing the number of transmissions has been deeply investigated by the Mobile Ad-hoc NETworks (MANETs) research community: the theoretically optimal solution consists in designating, as relays, the nodes belonging to the minimum connected dominant set (MCDS) of the network [2]. The nodes within the MCDS have the following properties: (i) they form a connected graph; (ii) every other node of the network is one-hop connected with a node in the MCDS; (iii) the MCDS has the lowest cardinality 
over all the possible collections of nodes that satisfy the previous two requirements.

Following the "idealized" MCDS-based design approach, a plethora of multihop broadcast protocols have been recently proposed in the VANET literature. Some of them, such as the emergency message dissemination for vehicular environments (EMDV) protocol [3], achieve remarkable performance by exploiting partial or complete knowledge of the network topology [4]. However, since collecting this information may be very expensive in terms of overhead, other techniques (requiring a reduced information exchange) have been proposed. An efficient IEEE 802.11-based protocol, denoted as urban multihop broadcast (UMB), was proposed in [5] and further extended in [6]. UMB suppresses the broadcast redundancy by means of a blackburst contention approach [7], followed by a ready-tosend/clear-to-send (RTS/CTS)-like mechanism. According to this protocol, a node can broadcast a packet only after having secured channel control. A different approach is adopted by another IEEE 802.11-based protocol, denoted as smart broadcast (SB) [8]. Similarly to $\mathrm{UMB}, \mathrm{SB}$ partitions the transmission range of the source, associating non-overlapping contention windows to different regions. The binary partition assisted protocol (BPAB) [9] uses concepts from both $\mathrm{UMB}$ and $\mathrm{SB}$, thus presenting similar performance, with an improvement, with respect to the SB protocol, in VANETs with low vehicle spatial density and irregular topologies. Finally, a different approach is considered when analyzing the class of probabilistic broadcast protocols, designed around the idea that each node forwards a received packet according to a characteristic probability assignment function (PAF), computed by each node in a distributed manner $[10,11]$. An entire class of probabilistic broadcast protocols is proposed and analyzed in [12].

In one-dimensional networks, as those considered in this work, knowledge of inter-node distances is necessary to implement the MCDS solution. For this reason, most of the proposed multihop broadcast protocols assume, at least to some extent, this knowledge. Therefore, the first step for deriving an analytical model consists in statistically characterizing the spatial distribution of the vehicles. In the literature, the node positions are frequently generated with a poisson point process (PPP), that allows to accurately model the real characteristics of the road topology. Despite its apparent simplicity, the derivation of an analytical performance evaluation framework based on the assumption of Poisson spatial distribution of the vehicles is not straightforward.

This work is motivated by the need of having a low complexity theoretical framework, useful for characterizing the main performance metrics of a family of probabilistic multihop broadcast protocols with applications to VANET scenarios. First, we show that the average positions of a given number of points of a PPP falling in a segment with finite length are equally spaced. Then, assuming a silencing mechanism at each hop, we derive a recursive (hop-wise) theoretical performance evaluation framework which exploits the assumption of fixed and equally spaced vehicles positions in each retransmission hop. In particular, this performance analysis is likely to be representative of the average (with respect to the nodes' spatial distribution) performance of the broadcast protocols at hand, as will be confirmed by ns2 simulations. Moreover, the proposed analytical model applies also to other vehicle spatial distributions, provided that the average inter-vehicle distance is fixed. The impact of node mobility will also be evaluated. Although we consider two novel illustrative broadcast protocols, we underline that our approach is general.

This article is structured as follows. In Section 2, multihop broadcast protocols for linear networks are introduced. Section 3 is devoted to the derivation of the average distribution of a given number of points of a PPP in a segment with finite length. In Section 4, a succinct overview of the IEEE $802.11 \mathrm{~b}$ standard is provided. In Section 5, the family of probabilistic broadcast protocol with silencing is accurately described. In Section 6, an analytical framework for performance evaluation of the probabilistic broadcast protocols of interest, is presented. In Section 7, after the validation of the analytical framework by means of numerical simulation, the performance of the novel probabilistic broadcast protocols is investigated and compared with that of other (known) protocols. Finally, Section 8 concludes the article.

\section{Multihop broadcast protocols}

\subsection{Reference scenario}

Figure 1 shows the linear network topology of reference for a generic multihop broadcast protocol: a static onedimensional wireless network with a source and $N$ (receiving) nodes. The assumption of static nodes is not restricting. In fact, from the perspective of a single transmitted packet, because of the very short transmission time (with typical IEEE 802.11 transmission rates), the network appears as static [13]. At the same time, a one-dimensional network is suitable for analyzing

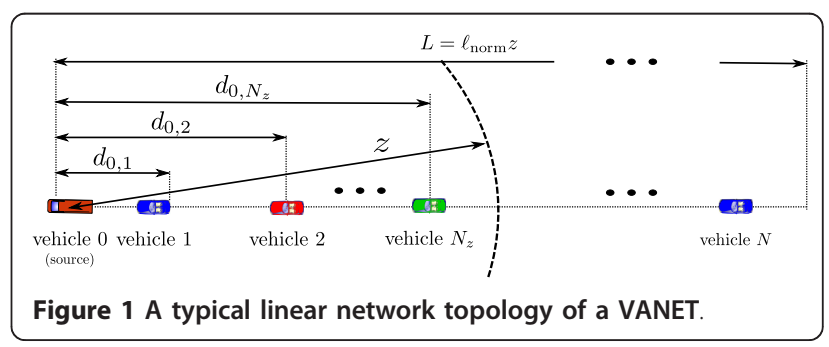


highway-like VANETs, where the width of the road (lying in the interval [10-40 m]) is significantly smaller than the transmission range of an IEEE 802.11 network interface. These motivations will be justified by simulation results in Section 7.

We consider a deterministic free-space propagation model (i.e., without fading) and a fixed transmit power: therefore, each vehicle has a fixed transmission range, denoted as $z$ (dimension: [m]). The network size (the line length) is set to $L$ (dimension: $[\mathrm{m}]$ ). For generality, we denote as normalized network size the positive real number $\ell_{\text {norm }} \triangleq L / z$. Generally, $\ell_{\text {norm }}>1$ and this motivates the need for multihop communication protocols.

On the basis of empirical traffic data [14], the nodes' positions are generated according to a PPP of parameter $\rho_{\mathrm{s}}$, where $\rho_{\mathrm{s}}$ is the vehicle (linear) spatial density (dimension: $[\mathrm{veh} / \mathrm{m}]$ )-the symbol "veh" it is not a realistic unit of measure, but it will be used for the sake of clarity. Consequently, $N$ is a random variable characterized by a one-dimensional Poisson distribution with parameter $\rho_{\mathrm{s}} L$. Similarly, the random variable $N_{z}$, denoting the number of nodes lying in the transmission range of the source (e.g., within the interval $(0, z))$, has a Poisson distribution with parameter $\rho_{\mathrm{s}} z$. Thanks to the properties of the Poisson distribution, the inter-vehicle distance is exponentially distributed with parameter $\rho_{\mathrm{s}}$ and the (constant) average distance between two consecutive vehicles is $1 / \rho_{\mathrm{s}}$.

As shown in Figure 1, the source node, denoted as node 0 , is placed at the west end of the network, and we assume a single propagation direction (eastbound). Each of the remaining $N$ nodes is uniquely identified by an index $i \in\{1,2, \ldots, N\}$. The distance between the $i$-th and $j$-th nodes $(i, j \in\{1,2, \ldots, N\}, i \neq j)$ is denoted as $d_{i, j}$. Each vehicle can exactly estimate the value of $d_{i, j}$, thanks to the following assumptions: (i) the position of the source is a-priori known by every node; (ii) each vehicle knows its own position under the assumption of the presence (on board) of a global positioning system (GPS) receiver; (iii) each rebroadcaster inserts its own geographical coordinates within the packet.

In the (one-dimensional and with a single propagation direction) scenario described in Figure 1, the operational principle of a multihop broadcast protocol is quite simple. The initial transmission of a new packet from the source is denoted as the 0 -th hop transmission, while the source itself identifies the so-called 0-th transmission domain (TD). After the source transmission, the packet is then received by the $N_{z}$ source's neighbors, that are the potential rebroadcasters at the 1-st hop. Hence, their ensemble constitutes the 1-st TD. Each vehicle in the 1-st TD decides to forward the packet according to a PAF specified by the broadcast protocol. The use of silencing corresponds to the fact that the "fastest" retransmitter (among the set of those which have decided to retransmit) silences the others. Note that a collision may happen if at least two nodes of a TD retransmit simultaneously. The propagation process is therefore constituted by multiple packet retransmissions, that continue at most till the east end of the networkas will be clear in the following, with a probabilistic broadcasting protocol the retransmission process might terminate before reaching the end of the network.

\subsection{Performance metrics of interest}

In this work, the performance of probabilistic multihop broadcast protocols will be investigated using the following average metrics: (i) the REachability (RE), (ii) the transmission efficiency (TE), and (iii) the end-to-end delay (D). The RE (adimensional), originally introduced in [1], is the fraction of nodes that receive the source packet among the set of all reachable nodes. The cardinality of the set of the reachable nodes is denoted as $n_{\text {reach}}$, and can be expressed as $n_{\text {reach }}=\min \left(N, n^{*}\right)$, where $n^{*}$ is the minimum index such as the condition $d_{n}{ }^{*}, n_{*}+1>z$ is verified. This definition is necessary since in PPP scenarios, as those considered in this work, there can exist a pair of disconnected consecutive nodes $\left(n^{*}, n^{*}+1\right)$. The TE (adimensional) is defined as the ratio between the RE of a packet and the overall number of rebroadcast acts experienced during its transmission to the last reachable node. Finally, D (dim: $[\mathrm{ms}]$ ) is defined as the duration of the packet trip between the source and the last reachable node. We remark that only the packets received correctly at the $n_{\text {reach }}$-th node of the network are considered for the evaluation of D. Therefore, this definition of D corresponds to a worst case scenario.

Owing to the symmetry of the forwarding process, the entire network can be modeled on the basis of the (local) analysis of a single TD. Therefore, in Section 3 we focus on a single TD-the reasons behind this assumption will be better clarified in Section 5 .

\section{Average distribution of poisson points in a segment with finite length}

We now present a constructive definition of a PPP with parameter $\rho_{\mathrm{s}} \in \mathbb{R}^{+}$, directly inspired from the one presented in [15, Ch. 3]. Given a finite interval $(-T / 2, T / 2) \subset$ $\mathbb{R}$, place $n \in \mathbb{N}$ points in $(-T / 2, T / 2)$, under the constraint that $n / T=\rho_{\mathrm{s}}$. A PPP is obtained by letting $n \rightarrow$ $\infty$ and $T \rightarrow \infty$, under the constraint that $n / T$ remains equal to $\rho_{\mathrm{s}}$. A PPP has the following properties: (i) the distance between two consecutive points is a random variable with an exponential distribution with parameter $\rho_{\mathrm{s}}$; (ii) given $z \in \mathbb{R}^{+}$, the number of points falling in the 


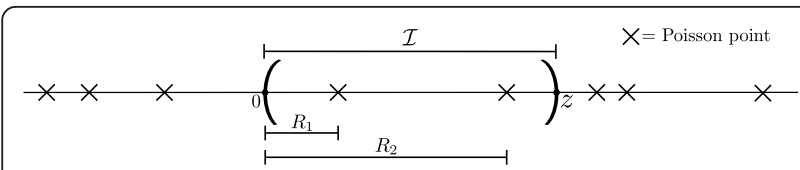

Figure 2 Illustrative realization of a PPP (the points corresponds to $\mathrm{X}$ )

finite interval $\mathcal{I} \triangleq(0, z) \subset \mathbb{R}$ is a random variable with a Poisson distribution with parameter $\rho_{\mathrm{s}} z$. In Figure 2, an illustrative realization of a PPP with parameter $\rho_{\mathrm{s}}$ is shown. With reference to Figure 2, denoting by $n$ the number of Poisson points falling in $\mathcal{I}$ it is possible to define the $n$-dimensional positions vector

$$
\mathbf{R}^{(n)}=\left[R_{1} R_{2} \ldots R_{n}\right]
$$

where $R_{i}(i \in\{1,2, \ldots, n\})$ is the distance of the $i$-th point from the source (placed in zero)-in the illustrative case in Figure 2, $n=2$.

In Appendix 1, it is shown that the marginal probability density function (PDF) of $R_{j}$ is the following:

$$
f_{R_{j}}^{(n)}(r)= \begin{cases}\frac{n !}{z^{n}} \frac{(z-r)^{n-j} r^{j-1}}{(n-j) !(j-1) !} & r \in(0, z) \quad j=1, \ldots, n \\ 0 & \text { otherwise. }\end{cases}
$$

In Figure 3, the PDFs of the positions of consecutive nodes are shown for various values of $n$ : (a) 1, (b) 2, and (c) 4. In Appendix 1, it is also shown that the average position of the $j$-th node can be expressed as follows:

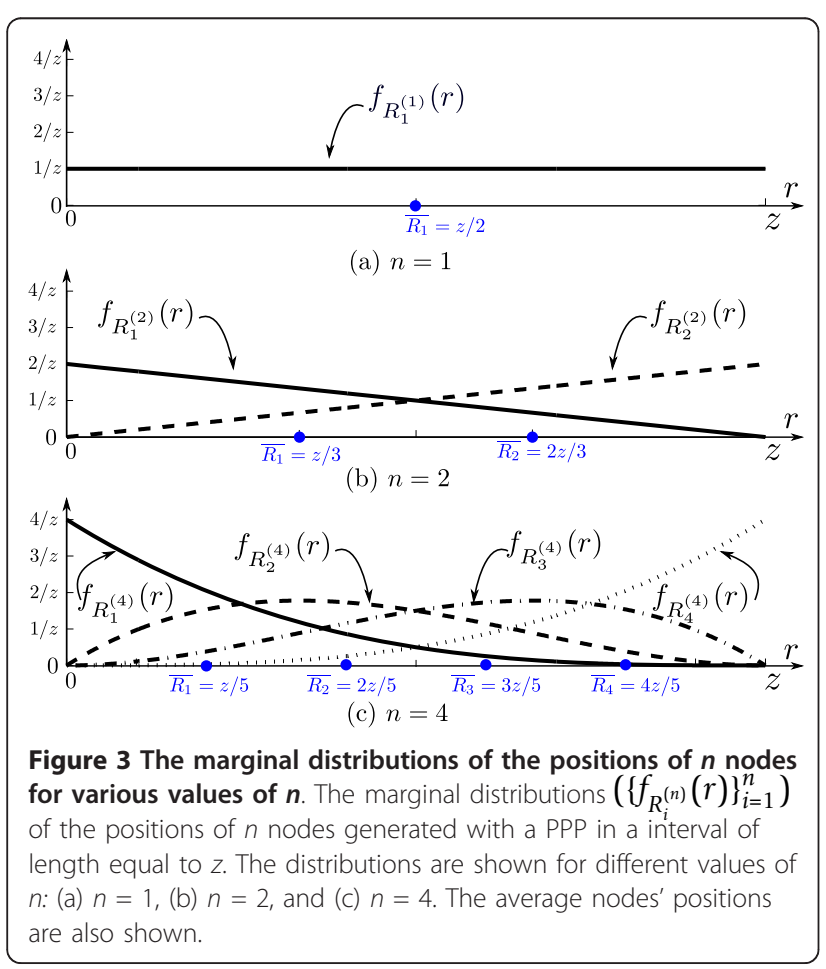

$$
\bar{R}_{j}^{(n)}=\int_{0}^{z} r \frac{n !}{z^{n}} \frac{(z-r)^{n-j} r^{j-1}}{(n-j) !(j-1) !} \mathrm{d} r_{j}=j \frac{z}{n+1} \quad j=1, \ldots, n .
$$

From Equation (3), it emerges clearly that, for a given number of nodes falling in a finite segment $\mathcal{I}$, their average positions are equally spaced. The average nodes' positions, for various values of the number $n$ of nodes in $\mathcal{I}$, are also shown in Figure 3.

Thanks to these results, the average performance analysis of a broadcast protocol in a network with Poisson node distribution can be carried out by simply studying a deterministic scenario, where the nodes are placed in correspondence to the average positions of the corresponding Poisson-based scenario. Moreover, this average analysis applies to other vehicle spatial distributions (e.g., taking into account the constraint on the vehicle lengths) with equally spaced average positions.

\section{A quick overview of the IEEE $802.11 \mathrm{~b}$ standard}

In this work, we assume that the physical and the medium access control (MAC) layers of every node adhere to the IEEE 802.11b standard [16]. In this section, we first recall the basic features of this standard. Due to the broadcast nature of the communications, the contention channel is managed through the basic access (BA) mechanism, the operational principle of which can be briefly described as follows. When a node has a frame ready to be transmitted, it checks if the channel remains idle for a period of time at least longer than a distributed interframe space (DIFS): if this is the case, the node is free to immediately transmit. On the opposite, if the wireless medium is busy, the node defers its transmission until the medium remains idle for a whole DIFS without interruption. In the latter case, once the DIFS has elapsed, the node generates a random backoff period, which corresponds to an additional waiting time before transmitting (pre-backoff). The node transmits when the backoff time has elapsed. At each transmission act, the backoff time is uniformly chosen in the range $[0, c w-1]$, where $c w$ is the current backoff window size, that is constant and equal to the minimum value defined by the standard, denoted as $C W_{\min }$, and corresponding to 32 . The backoff period is slotted and the duration of the backoff, expressed in terms of number of backoff slots, is denoted as backoff counter (BC). This number is decremented as long as the medium is sensed idle, and it is frozen when a transmission is detected on the channel (this is an instance of a collision avoidance mechanism). Decrementing restarts when the medium is sensed idle again for more than a DIFS. At the end of every packet transmission, the node is forced to enter a post-backoff phase that coincides 
with the subsequent pre-backoff if the node has another packet in the transmission queue.

It is important to observe that when a relay finds the channel idle, it can immediately transmit, but this is not mandatory. In order to reduce the number of collisions within a TD, we have interpreted the standard in a nonpersistent manner, imposing that every relay enters into the pre-backoff phase, regardless of the channel status. We also remark that the extension of our approach to scenarios with IEEE 802.11p [17] communications, as envisioned in VANETs, is straightforward. Our approach (based on the IEEE 802.11b standard) is meaningful under the assumption of smartphone-based vehicular communications $[18,19]$.

\section{Probabilistic broadcast protocols with silencing 5.1 Preliminaries considerations}

The general goal of a multihop broadcast protocol is to attain the widest network coverage in the shortest possible time. This can be obtained by pursuing three intermediate goals: (i) minimizing the number of communication hops; (ii) minimizing the number of effective retransmissions in every hop; (iii) minimizing the latency associated with a single hop. The number of transmission hops can be minimized by designating, as relays, the nodes forming the MCDS. However, the number of retransmissions and the latency are directly affected by the protocol characteristics, and there is no general rule for minimizing them-this motivates the presence, in the literature, of a large number of heuristic broadcast protocols.

A probabilistic broadcast protocol tries to achieve the goals outlined in the previous paragraph in a probabilistic and completely distributed manner: (i) probabilistic, in the sense that every intermediate node decides to retransmit a packet according to a certain PAF, computed on a per-packet manner-even if, in general, one could introduce a per-flow PAF, in this work we focus on single packet transmissions; (ii) distributed, in the sense that every node autonomously makes a retransmission decision without any coordination with its neighbors.

In "classical" probabilistic broadcast protocols (without silencing), without adopting suitable counter-measures it is possible that more than one node in a TD decides to rebroadcast the packet (even without collisions). This leads to inefficiencies-besides complicating the mathematical analysis. A more efficient probabilistic broadcast protocol, regardless of the expression of the PAF, is obtained in the presence of a single retransmitting node in every TD. This can be obtained by imposing that the reception of a packet sent by a node of a TD silences the preceding nodes of the same TD. As a consequence, the next TD starts from the node which follows the "silencer." Note that the last TD partially overlaps with the previous one if the "silencer" is not a member of the MCDS.

In this work, we consider two novel probabilistic broadcast protocols with silencing, whose operations can be described as follows, with respect to the first TD.

(1) The source sends a new packet (directly mapped on a IEEE 802.11 frame).

(2) The nodes within a distance $z$ from the source receive the packet and form the 1 -st TD. Their number is denoted as $N_{z}$.

(3) Every node in the 1-st TD probabilistically decides, according to the given PAF and taking into account its distance from the source, to retransmit (or not) the packet.

(4) The potential forwarders (i.e., the nodes of the 1st TD which have decided to retransmit) compete for channel access, by using the BA mechanism of the IEEE 802.11b standard (described in Section 4), first entering in the pre-backoff phase and, then, generating a random waiting time (denoted, in Section 4 , as BC). For the purpose of analytical simplicity, we assume that the $\mathrm{BCs}$ of the losing contenders are set to $\infty$.

(5) The BCs are continuously decreased by all nodes, until (in the case of a successful forwarding) only one of them reaches 0 , say the $k$-th $\mathrm{BC}$. During a transmission of a node the other BCs freeze. Should there be the BCs of at least two nodes which reach simultaneously zero, both nodes would transmit and, thus, collide. We assume that the packets involved in a collision are considered undetectable and ignored by the other nodes. The corresponding $k$-th node retransmits the packet.

(6) The remaining $N_{z}-1$ nodes decode the packets, reset their timers, and discard the potentially queued packet. The nodes (spatially) preceding the $k$-th node will refrain from retransmitting from then on.

(7) The whole process (from Step 1) is restarted at the 2-nd TD, for which the $k$-th node acts as the source. The 2-nd TD is composed by all nodes lying in the interval $\left(d_{0, k}, d_{0, k}+z\right) \subset \mathbb{R}$, and it can also include some former nodes of the 1-st TD (those following the $k$-th node).

The two novel probabilistic broadcast protocols, polynomial and SIF, are described in the following two subsections.

\subsection{Polynomial broadcast protocol}

This protocol is characterized by a polynomial PAF, with the following form: 


$$
p(d, z, g) \triangleq\left(\frac{d}{z}\right)^{g}
$$

where: $d$ denotes the distance (dimension: $[\mathrm{m}]$ ) between the node of interest and the previous relay (or source, in the case of the first TD); $z$ is the already introduced transmission range; $g \in \mathbb{N}$ is the polynomial order. According to the assumptions in Section 2, both $z$ and $d$ are assumed to be known without the need of exchanging additional messages. In fact, $z$ can be estimated by knowing the transmit power and the channel propagation model, while $d$ can be estimated by simply inserting the position of the source vehicle in every transmitted packet (under the assumption of having an accurate GPS receiver).

The shape of $p$, as a function of $d$, is shown in Figure 4 , for different values of $g$. It can be observed that the function $p$ is monotonic and concave for all values of $g$. For high values of $g$, it becomes quite "selective," since it is approximately zero everywhere, but in the proximity of $z$. Note that the case with $g=0(p=1, \forall d)$ corresponds to the flooding protocol, i.e., each node retransmits. In this case, the $\mathrm{BC}$ value is randomly selected in $\{0,1, \ldots, c w-1\}$ as mandated by the IEEE 802.11 standard (Section 4).

\subsection{Silencing irresponsible forwarding}

This broadcast protocol directly derives from the irresponsible forwarding (IF) protocol, originally presented in [20], with the introduction of the silencing mechanism with the introduction of the silencing mechanism outlined in Section 5.2. Besides this difference, IF and

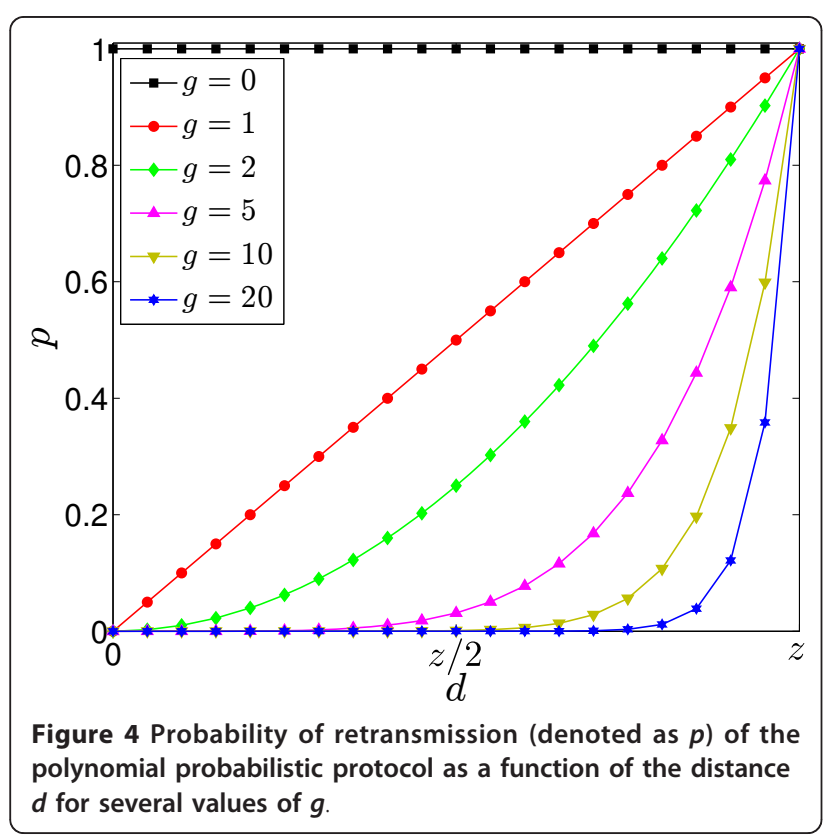

SIF share the same following PAF:

$$
p(d, z, g) \triangleq \exp \left\{-\rho_{s} \frac{(d-z)}{c}\right\}
$$

where $c$ is an adimensional shaping coefficient and $\rho_{\mathrm{s}}$ is the vehicle spatial density. The latter can be estimated in a straightforward manner. In fact, under the assumption of knowing with a sufficient accuracy its transmission range, a node can estimate its local vehicular spatial density by simply counting the number of nodes lying within its transmission range and dividing them by the transmission range. The design of an efficient method for accurate estimation of the vehicular spatial density goes beyond the scope of this manuscript. However, intuitively it is sufficient to periodically send (and receive) Hello messages to the surrounding nodes. Alternatively, it is possible to rely on already existing beaconing mechanisms, such as the exchange of cooperative awareness messages (CAMs) foreseen by the European car-to-car consortium (broadcasted by default every 500 $\mathrm{ms})$ [21].

Similarly to the PAF of the polynomial broadcast protocol, also the PAF of SIF "rewards" the farthest nodes (with respect to the transmitter). However, unlike the polynomial PAF, the PAF of SIF also takes into accounts the (linear) vehicular spatial density, thus allowing to better adapt to different traffic conditions- this is the very idea of IF. The shape of $p$, as a function of $d$, is shown in Figure 5, for different values of $c$ and $\rho_{\mathrm{s}}$. It can be observed that the PAF of SIF is monotonically increasing and concave for all values of $c$. Moreover, it becomes selective far small values of $c$ (e.g., 1), while it tends to flatten for high values of $c$ and for low values of $\rho_{\mathrm{s}}$. Also in this case, the BC value is randomly

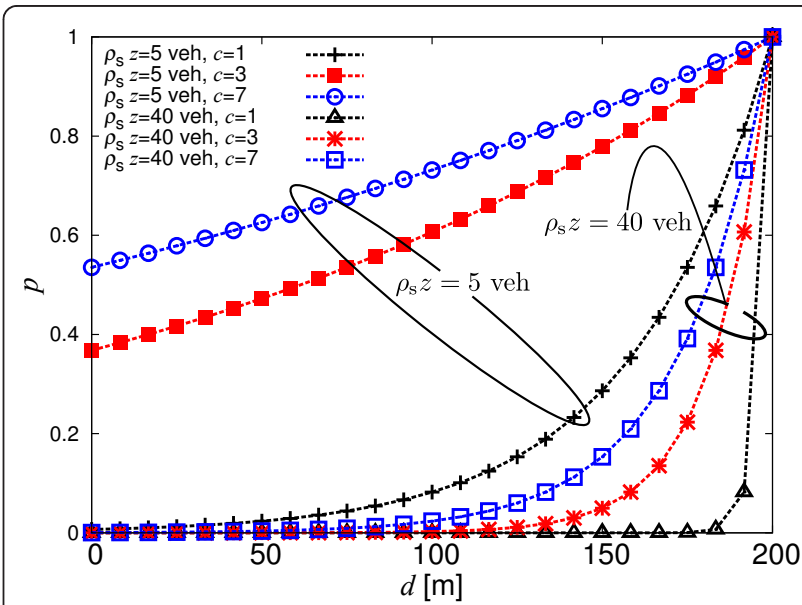

Figure 5 Probability of retransmission (denoted as $p$ ) of the SIF protocol as a function of the distance $d$ for several values of $c$ and $\rho_{\mathrm{s}} z$. 
selected in $\{0,1, \ldots, c w-1\}$ as mandated by the IEEE 802.11 standard (Section 4).

\section{A recursive analytical performance evaluation framework}

In Section 2, it has been stated that, since all TDs are statistically identical, the global behavior of the network can be modeled by analyzing a single TD. By exploiting the properties of probabilistic broadcast protocols with silencing (described in Section 5), the following assumptions hold: (i) the inter-node distance is characterized by a (memoryless) exponential distribution, so that the topology of every TD is (statistically) identical; (ii) the PAF only depends on the distance and is, therefore, memoryless; (iii) the IEEE $802.11 \mathrm{~b}$ contention mechanism is memoryless, in the sense that it is restarted at every retransmission. Under these assumptions, every retransmission act can be interpreted as a renewal that resets the statistics of the forwarding process. Moreover, since all TDs are statistically identical, without loss of generality we can focus on the first TD.

Therefore, a complete analytical performance evaluation framework can be derived in the following manner: (i) characterizing the first TD with local performance metrics (e.g., the successful transmission probability and the delay); (ii) deriving global performance metrics (e.g., $\mathrm{D}, \mathrm{RE}, \mathrm{TE})$, by means of a recursive approach.

In Section 6.1, the local performance (i.e., single TD) is investigated under the assumption of a given number of equally spaced nodes, by considering, without loss of generality, the first TD. In Section 6.2, we derive the global metrics for an overall deterministic network scenario, where the nodes are equally spaced in the interval $(0, L)$. Then, in Section 6.3 the results obtained in the deterministic scenario are extended to the original PPPbased scenario.

\subsection{Local (single TD) performance analysis with a given number of nodes}

Without loss of generality, we focus on the first TD, corresponding to the interval $\mathcal{I}$ introduced in Section 3. We consider a deterministic scenario with a fixed number $n$ of nodes equally spaced in the interval $\mathcal{I}=(0, z) \subset \mathbb{R}$. Every node in a TD is identified by an index $i \in\{1,2, \ldots, n\}$. The nodes are thus positioned as in Figure 3 and the positions vector $\mathbf{R}^{(n)}$ is defined as in (1).

According to the operational principles of the considered protocol, after the reception of a packet in a given TD, each node decides to (or not to) retransmit according to the protocol's PAF. The nodes that lose the contention set their BCs to $\infty$, while the winners set their $\mathrm{BCs}$ according to the policy of the specific broadcast protocol. The protocol execution could lead to three different outcomes: (i) nobody decides to retransmit; (ii) some nodes decide to retransmit, but all their transmitted packets collide; (iii) some nodes decide to retransmit, and a single node transmits successfully (when its $\mathrm{BC}$ because zero, no other $\mathrm{BC}$ is zero). It is useful to define the following events, associated to the forwarding process in a TD:

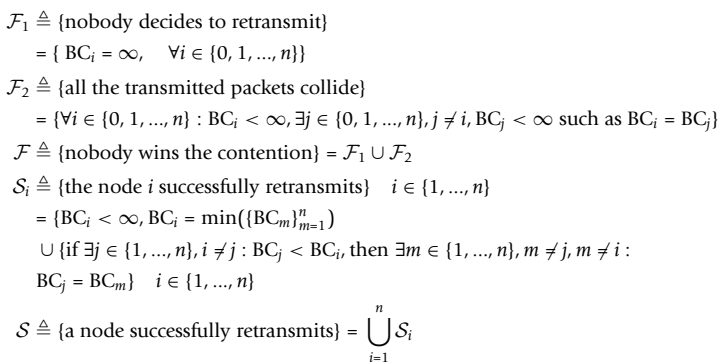

The probabilities of the above defined events are the following:

$$
\begin{aligned}
& p_{\mathrm{rtx}}^{(n)}(i) \triangleq \mathrm{P}\left\{\mathcal{S}_{i}\right\} \quad i=1,2, \ldots, n \\
& p_{\text {succ }}^{(n)} \triangleq \mathrm{P}\{\mathcal{S}\}=\sum_{i=1}^{n} p_{\mathrm{rtx}}^{(n)}(i) \\
& p_{\text {fail }}^{(n)} \triangleq 1-P\{\mathcal{S}\}=1-\sum_{i=1}^{n} p_{\mathrm{rtx}}^{(n)}(i) .
\end{aligned}
$$

Let us now introduce the random variable $Y \in\{0,1$, $2, \ldots, n\}$ with the following PMF:

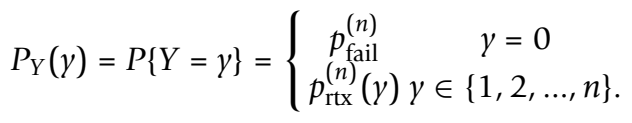

Since the event $\{Y=0\}$ identifies the failure event, the random variable $Y$ indicates either which node has effectively retransmitted or a failure. Moreover, it can be observed that:

$$
\bigcup_{\gamma=1}^{n}\{Y=\gamma\}=\mathcal{F} \cup \mathcal{S} .
$$

Obviously,

$$
P_{Y}(\gamma \mid \mathcal{S})=P_{Y}(Y=\gamma \mid \mathcal{S})=\left\{\begin{array}{cc}
0 & y=0 \\
\frac{p_{\mathrm{rtx}}^{(n)}(x)}{\sum_{t=1}^{n} p_{\mathrm{rtx}}^{(n)}(i)} y \in\{1,2, \ldots, n\} .
\end{array}\right.
$$

In other words, if there is a retransmission $(\mathcal{S})$, then $P_{Y}(y \mid \mathcal{S})(y \in\{0,1,2, \ldots, n\})$ is the probability that the $y$ th node has retransmitted.

As shown in Appendix 2, the transmission probabilities $\left\{p_{\mathrm{rtx}}^{(n)}(i)\right\}$ can be expressed as follows: 


$$
p_{\mathrm{rtx}}^{(n)}(i)=p_{i} \sum_{m=1}^{n} q^{(m)} p_{V_{i}^{(n)}}(m-1)
$$

where: $p_{i}$ denotes the value of the PAF (4) for the $i$-th node and depends on the considered protocol; $q^{(m)}$ is the probability that the $i$-th node wins the contention among a set of $m$ competing nodes (the same for a given value of $n) ; V_{i}^{(n)} \in\{0, \ldots, n-1\}$ is the following discrete random variable:

$$
V_{i}^{(n)} \triangleq \text { number of nodes, among the } n \text { nodes, competing with the } i-\text { th node }
$$

The derivation of $q^{(m)}$ and of the PMF of $V_{i}^{(n)}$ can also be found in Appendix 2.

After deriving $p_{\mathrm{rtx}}^{(n)}(i)$, it is possible to compute the per-hop delay, denoted as $D_{i}$, of a retransmission from the $i$-th node. Since the per-hop delay is meaningful only if the $i$-th node decides to retransmit, it is of interest to study the statistical distribution of $D_{i}$ conditioned on $S_{i}$. For this reason, we introduce the random variable $D_{i \mid i}$, which can be defined as follows:

$$
D_{i \mid i} \triangleq T_{\text {slot }}\left(D I F S+N_{i \mid i}^{\mathrm{bo}}\right)+T^{\mathrm{tx}} \quad i=1, \ldots, n
$$

where: $T^{\mathrm{tx}}$ (dimension: [s]) is the transmission time; $T_{\text {slot }}$ (dimension: [s/slot]) is the deterministic duration of the backoff slot; DIFS (dimension: [slot]) is the duration of the DIFS; and $N_{i \mid i}^{\text {bo }}$ (dimension: [slots]) is the number of slots spent by the $i$-th node during the backoff (conditionally on the event $S_{i}$ ). We assume that both the packet size, defined as $P$ (dimension: [bits]), and the transmission rate, denoted as $R$ (dimension: [bits/s]), are constant, thus leading to a deterministic packet transmission time $T^{\mathrm{tx}}=P / R$. Taking into account that DIFS, $T_{\text {slot }}$, and $T^{\mathrm{tx}}$ are deterministic, the average value of $D_{i \mid i}$ becomes:

$$
\bar{D}_{i \mid i}=T_{\text {slot }}\left(D I F S+\bar{N}_{i \mid i}^{\mathrm{bo}}\right)+T^{\mathrm{tx}} \quad i=1, \ldots, n
$$

where, according to the derivation in Appendix 3,

$$
\bar{N}_{i \mid i}^{b o}=\frac{p_{i}}{c w p_{\mathrm{rxx}}^{(N)}(i)} \sum_{v=0}^{N-1} p_{V_{i}}^{(N)}(v) \sum_{k=1}^{c w-1}\left[k \sum_{j=0}^{J_{k, v}} P_{v}^{\prime}(k, j)+T^{\mathrm{tx}} \sum_{j=1}^{I_{k, v}} j P_{v}^{\prime}(k, j)\right]
$$

where $J_{k, v} \triangleq \min (k,\lfloor(v / 2)\rfloor)$ denotes the maximum number of collisions that can happen in slots $0,1, \ldots, k$ 1 , while the matrix $\mathbf{P}_{v}^{\prime}=\left\{P_{v}(k, j)\right\}$ is defined in Appendix 3.

Proceeding in a similar manner, it is also possible to obtain the average number of retransmissions per-hop of the node $i$, denoted as $\bar{N}_{\mathrm{rtx}}^{\text {hop }}(i)$ :

$$
\bar{N}_{\mathrm{rxx}}^{\mathrm{hop}}(i)=\frac{p_{i}}{c w p_{\mathrm{rtx}}^{(N)}(i)}\left(1+\sum_{v=0}^{N-1} p_{V_{i}^{(N)}}(v) \sum_{k=1}^{c w-1} \sum_{h=2}^{v} h N_{k, v}(0, h) \sum_{j=0}^{J_{k, v}} M_{k, \nu}(j, h)\right)
$$

where the matrices $\mathrm{M}_{k, v}=M_{k, v}(j, h)$ and $\mathrm{N}_{k, v}=N_{k, v}(j$, h) are defined in Appendix 3.

\subsection{Global performance analysis with fixed number of nodes}

Once the per-TD performance has been analyzed (as described in Section 6.1), the global performance metrics introduced in Section 2.2 (namely, RE, TE, and D) can be computed by following a recursive approach, based on the inductive principle. This recursive approach is extensively described, for the evaluation of $\mathrm{D}$, in Appendix 4, but can be directly re-adapted for the evaluation of RE and TE. In the remainder of this subsection, we outline the final results, trying to provide the reader with the intuition behind them.

Recall that we consider a deterministic scenario with a fixed number $N$ of nodes equally spaced in the interval $(0, L) \subset \mathbb{R}$, where $L=z \ell_{\text {norm }}$. For simplicity, we assume that a generic TD contains $n=N / \ell_{\text {norm }}$ nodes. This corresponds to a best-case scenario, where the farthest node of each TD is the domain forwarder (the "silencer," as denoted in Section 5).

Delay The computation of the average D is carried out taking into account only the packets successfully arriving at the end of the network (i.e., at the last reachable node) and ignoring the (remaining) packets which stop earlier. On the basis of the approach described in detail in Appendix 4, the average end-to-end delay can be given the following recursive formulation:

$$
D \triangleq \bar{D}^{(N)}=\bar{T}_{\mathrm{src}}^{\mathrm{tx}}+\sum_{i=1}^{n}\left(\bar{D}^{(N-i)}+\bar{D}_{i \mid i}\right) p_{Y}(i \mid \mathcal{S})
$$

where $\bar{D}^{(N-i)}$ is the average delay in a network with $N$ - $i$ nodes and $\bar{T}_{\mathrm{src}}^{\mathrm{tx}}$ is the average transmission time of the source, which differs from those of the following nodes, since the source does not contend with any other node and its transmission is not affected by collisions. Since the average time spent in the backoff is $\bar{T}_{\text {src }}^{\mathrm{tx}}$ can be expressed as

$$
\bar{T}_{\mathrm{src}}^{\mathrm{tx}} \triangleq T^{\mathrm{tx}}+T_{\text {slot }}\left(D I F S+\frac{c w-1}{2}\right) .
$$

$R E$ The average RE can be defined as follows:

$$
\mathrm{RE} \triangleq \frac{\overline{N_{\text {reach }}}}{N}
$$


where $N_{\text {reach }}$ is a random variable denoting the number of nodes reached by a packet. As a consequence of our assumptions, $N_{\text {reach }}$ is lower bounded by $n$, since the transmission from the source reaches $n$ nodes (those of the first TD) with probability 1 . The average value $\bar{N}_{\text {reach }}$ can be obtained by following the approach described in Appendix 4, but for the replacement of $p_{Y}(i \mid \mathcal{S})$ with $p_{\mathrm{Y}}(i)$ and of $\bar{D}_{i \mid i}$ with the number of additional nodes covered by a new transmission. For example: a transmission from the 1st node of the first TD will reach only one additional node (namely, the $(n+1)$-th); a transmission from the 3 -rd node will reach three additional nodes (namely, the $(n+1)$-th, $(n+2)$-th, and $(n+3)$-th); and so on. Please note that, unlike the delay, in the computation of the RE we are not conditioning on the fact of reaching the $\mathrm{N}$-th node of the network, i.e., the last reachable node of the network. Therefore, also the packets which stop being retransmitted are taken into account.

After the execution of the recursive approach outlined in Appendix 4, it is sufficient to add a constant equal to $n$, corresponding to the number of nodes directly reached by the source at the first hop. The final expression of $\bar{N}_{\text {reach }}$ becomes (using the notation of Appendix $4)$ :

$$
\begin{aligned}
\bar{N}_{\text {reach }} & =\bar{N}_{\text {reach }}^{(N)}=n+\sum_{i=1}^{n}\left(\bar{N}_{\text {reach }}^{(N-i)}+i\right) p_{Y}(i) \\
& =n+\sum_{i=1}^{n}\left(\bar{N}_{\text {reach }}^{(N-i)}+i\right) p_{\text {rtx }}^{(n)}(i)
\end{aligned}
$$

where $\bar{N}_{\text {reach }}^{(N-i)}$ corresponds to the average number of nodes reached in a network with $N-i$ nodes and can be recursively computed in the same way.

$T E$ In order to reduce the computational burden, we adopt the following approximated formulation of TE:

$$
\mathrm{TE} \triangleq \frac{\mathrm{RE}}{N_{\mathrm{rtx}}}
$$

where $\bar{N}_{\text {rtx }}$ denotes the average overall number of retransmissions over all hops. From a computation viewpoint $\bar{N}_{\mathrm{rtx}}$ is approximated by $\bar{N}_{\mathrm{rtx}}^{m^{(*)}}$, where $m^{*}$ corresponds to the average number of reached nodes-it is a sort of approximated indicator of the "depth" of the propagation process. Since the RE can be interpreted as the ratio between the average number of reached nodes and the total number $(N)$ of nodes, $m^{*}$ can be approximated as follows:

$$
m * \simeq N \cdot \mathrm{RE} .
$$

At this point, $\bar{N}_{\mathrm{rtx}}^{m^{(*)}}$ can be computed by applying the recursive approach presented in Appendix 4, by replacing (i) $p_{Y}(i \mid \mathcal{S})$ with $p_{Y}(i)$ and (ii) $\bar{D}_{i \mid i}$ with the average number of transmissions per hop, denoted by $\bar{N}_{\mathrm{rtx}}^{\text {hop }}$ and given in (9).

\subsection{Generalization to a PPP-based scenario}

According to the original PPP-based model, described in Section 2, the number of nodes within $\mathcal{I}$, denoted as $N_{z}$, has the following Poisson distribution:

$$
p_{N_{z}}\left(n, \rho_{\mathrm{s}} z\right)=\frac{e^{-\rho_{\mathrm{s}} z}\left(\rho_{\mathrm{s}} z\right)^{n}}{n !} n \in\{0,1,2, \ldots\} .
$$

However, since a real vehicle has a finite length, it is not possible to have an infinite number of vehicles within $\mathcal{I}$. Therefore, it makes sense to impose an arbitrary limit to the maximum number of nodes within $\mathcal{I}$, denoted as $N_{c}$. The new truncated Poisson random variable, denoted as $N_{z}^{\prime}$, has the following distribution:

$$
p_{N^{\prime} z}\left(n, \rho_{\mathrm{s}} z\right)=\frac{\frac{e^{-\rho_{\mathrm{s}} z}\left(\rho_{\mathrm{s}} z\right)^{n}}{n !}}{\sum_{i=1}^{N_{c}} \frac{e^{-\rho_{\mathrm{s}} z}\left(\rho_{\mathrm{s}} z\right)^{i}}{i !}} \quad n \in\left\{1,2, \ldots, N_{c}\right\}
$$

where we have also removed the event $n=0$-this would correspond to an empty TD.

In order to exploit the results of Section 6.1, the stochastic network topology of the PPP needs to be mapped into a deterministic one with equally spaced nodes. In order to do this, the interval $\mathcal{I}$ is partitioned in $N^{\text {int }}$ sub-intervals of length $z / N^{\text {int }}$, where $N^{\text {int }} \in\left\{N_{c}\right.$, $\left.N_{c}+1, N_{c}+2, \ldots\right\}$ is a design parameter. The computational burden and the accuracy are directly related to the value of $N^{\text {int }}$. After some numerical tests, we observed that the value $N^{\text {int }}=100$ is a good tradeoff between precision and computational time. The $i$-th sub-interval thus is:

$$
\mathcal{I}_{i}=\left[\frac{(i-i) z}{N^{\mathrm{int}}}, \frac{i z}{N^{\mathrm{int}}}\right] \quad i=1,2, \ldots, N^{\mathrm{int}} .
$$

Every sub-interval can contain at most one node: in general, we assume that in each sub-interval there is a "virtual" node. Consequently, it is possible to associate a transmission probability $p_{\mathrm{rtx}}^{\mathrm{eq}}(i)$ to the generic sub-interval $\mathcal{I}_{i}$, defined as $p_{\mathrm{rtx}}^{\text {eq }}(i)$, and a corresponding per-node delay, denoted as $D(i)^{\text {eq }}\left(i=1, \ldots, N^{\text {int }}\right)$.

We define as $p_{\mathrm{rtx}}^{(n)}(j)$ the probability of retransmission of the $j$-th node, given that there are exactly $n$ nodes in the interval $\mathcal{I}$. Using the total probability theorem, $p_{\mathrm{rtx}}^{\mathrm{eq}}(i)$ can be expressed as follows: 


$$
\begin{aligned}
p_{\mathrm{rtx}}^{\mathrm{eq}}(i) & =\sum_{n=1}^{N_{c}}\left(p_{\mathrm{rtx}}^{\mathrm{eq}}(i) \mid N_{z}^{\prime}=n\right) P\left(N_{z}^{\prime}=n\right) \\
& =\sum_{n=1}^{N_{c}} \sum_{j=1}^{n} p_{\mathrm{rtx}}^{(n)}(j) f(i, j, n) p_{N_{z}^{\prime}}\left(n, \rho_{\mathrm{s}} z\right) i \in\left\{1, \ldots, N^{\mathrm{int}}\right\}
\end{aligned}
$$

where $f(i, j, n)$ is an indicator function defined as follows:

$$
f(i, j, n) \triangleq\left\{\begin{array}{l}
1 \overline{\mathrm{R}}_{j}^{(n)} \in \mathcal{I}_{i} \\
0 \overline{\mathrm{R}}_{j}^{(n)} \notin \mathcal{I}_{i} .
\end{array}\right.
$$

The probability $p_{\mathrm{rtx}}^{\mathrm{eq}}(i)$ is now a function of $p_{\mathrm{rtx}}^{(n)}(i)(n \in$ $\left.\left\{1,2, \ldots, N_{c}\right\}, i \in\{1,2, \ldots, \mathrm{n}\}\right)$, which can be computed with combinatorics, since it is associated with a deterministic scenario with $n$ static nodes equally spaced in [0, $z]$.

At this point, by using (6) in Equation (15), it is possible to obtain a closed-form expression for $p_{\mathrm{rtx}}^{\mathrm{eq}}(i)$. Leveraging on the knowledge of $p_{\mathrm{rtx}}^{\mathrm{eq}}(i)$, by using Equations (15) into (7) and (9), it is possible to obtain, respectively, $D(i)^{\text {eq }}\left(i=1, \ldots, N^{\text {int }}\right)$ and $n_{\mathrm{rtx}}^{\text {hop }}$. Then, it is possible to use the framework presented in Section 6.2 to derive RE, $\mathrm{TE}$, and $\mathrm{D}$ for a deterministic network composed by $N_{c} \ell_{\text {norm }}$ nodes, since $N_{c}$ is the (imposed) number of nodes in the interval $\mathcal{I}$ (and, thus, in each TD).

As anticipated at the end of Section 1, we remark that the presented analytical framework can be employed to study other types of broadcast protocols, not necessarily probabilistic, by simply re-adapting the definition of $p_{\mathrm{rtx}}^{(n)}(i)$ and $D_{i \mid i}$. This is the subject of our current research activities.

\section{Theoretical performance analysis and simulation-based validation \\ 7.1 Polynomial protocol}

In this section, we compare the results obtained with the analytical framework presented in Section 6 with results obtained through numerical simulations carried out with the ns-2.34 simulator [22]. In particular, the polynomial protocol has been "inserted" on top of the IEEE $802.11 \mathrm{~b}$ model, after fixing the bugs reported in [23]. We observe that, conditionally on the fact of suitably scaling the packet size and the packet generation rate, from the perspective of our framework the IEEE $802.11 \mathrm{a} / \mathrm{p}$ standards will offer the same performance of the IEEE $802.11 \mathrm{~b}$ standard. All the results presented are accurate within $\pm 5 \%$ of the values shown with $95 \%$ confidence. The relevant parameters of the simulation are listed in Table 1. The results are obtained for a fixed node spatial density $\rho_{\mathrm{s}}=0.1 \mathrm{veh} / \mathrm{m}$, while the possible values of the transmission range $z$ are listed in Table 1 . In particular, the values of $z$ are selected so that the
Table 1 Main IEEE $\mathbf{8 0 2 . 1 1 b}$ network simulation

\begin{tabular}{ll}
\multicolumn{2}{l}{ parameters } \\
\hline IEEE $802.11 \mathbf{b}$ simulation \\
\hline$\rho_{\mathrm{s}}$ & $0.1 \mathrm{veh} / \mathrm{m}$ \\
$z$ & $\{100,150,200,300,400\} \mathrm{m}$ \\
$\ell_{\text {norm }}$ & 8 \\
Packet size & 1,000 bytes \\
Carrier freq. & $2.4 \mathrm{GHz}$ \\
Data rate & $1 \mathrm{Mbps}$ \\
$C W_{\text {MIN }}$ & 31 \\
\hline
\end{tabular}

corresponding values of $\rho_{\mathrm{s}} z$ are between 10 and 40veh. In the numerical simulations, we do not consider any case with $\rho_{\mathrm{s}} z<10$ veh, since this corresponds to topologies that are disconnected with a high probability, as shown in [10]. In Figure 6, (a) D, (b) RE, and (c) TE are shown as functions of $\rho_{s} z$, for different values of $g$, by taking into account both the results of the analytical framework and of the numerical simulations, thus allowing to assess the validity of the analytical model. As shown in [10], using the considered values of $\rho_{s} z$ (between 10 and 40 veh), the network is fully connected (i.e., $n_{\text {reach }}=$ $N)$ with a high probability. From Figure $6 \mathrm{~b}$ it emerges that, in terms of RE, there is an excellent match between the results of the theoretical framework and those of the simulator. As shown by Figure 6c, the agreement between analysis and simulations is still good also in terms of TE. On the other hand, the delay predicted by the analytical framework overestimates the true delay for small values of $g$ (e.g., $g=0$ ), whereas it becomes very accurate for large values of $g$ (e.g., $g=7$ ). The comparative investigation of analytical and simulation results indicates the validity of the proposed framework (especially for large values of $g$ ).

According to the results in Figure 6a,c, it emerges that a higher polynomial degree leads to a better performance, regardless of the value of $\rho_{\mathrm{s}} z$, in terms of both $\mathrm{D}$ and TE. Conversely, since the PAF is highly selective for large values of $g$ (as shown in Figure 4), this leads to poor performance in terms of RE, as shown in Figure 6b. By considering small values of $g$ (e.g., $g=0$ corresponds to flooding), one observes the opposite phenomenon: a drastic improvement in terms of RE, at the price of a slightly higher D and a smaller TE.

In order to better understand the impact of $g$ and $\rho_{\mathrm{s}} z$ on the protocol performance: in Figure 7a, D is shown, parametrized with respect to $g$, as a function of RE for different values of $\rho_{\mathrm{s}} z$; while in Figure 7b D is shown, parametrized with respect to $\rho_{\mathrm{s}} z$, as a function of RE for different values of $g$. From the results in Figure $7 a$, it emerges that even little variations of $g$ lead to radically different protocol behaviors. On the contrary, $\rho_{\mathrm{s}} z$ has an impact on the performance only for small values of $\rho_{\mathrm{s}} z$, 


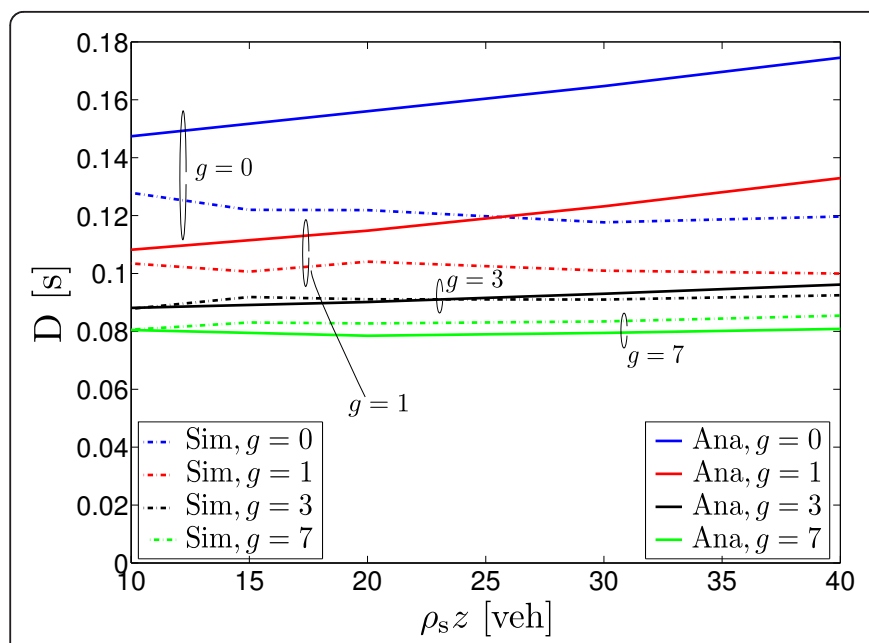

(a)

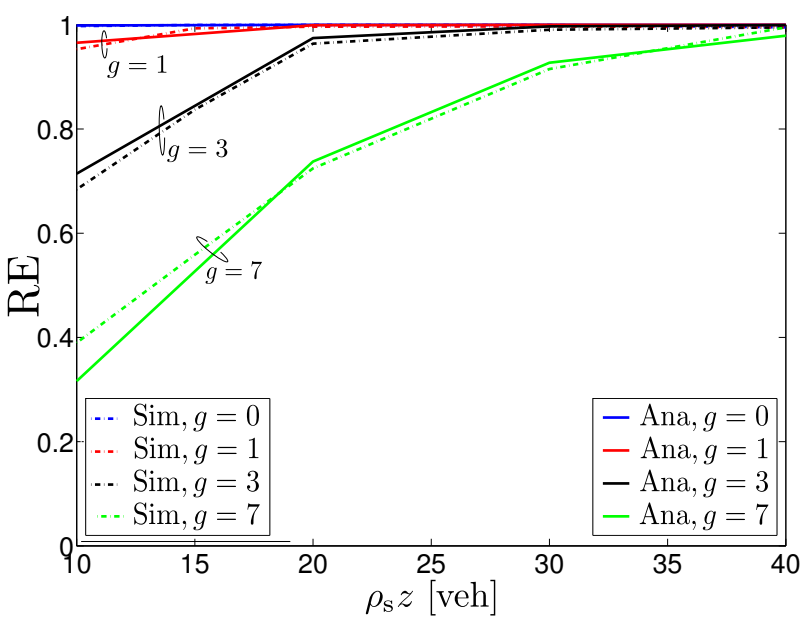

(b)

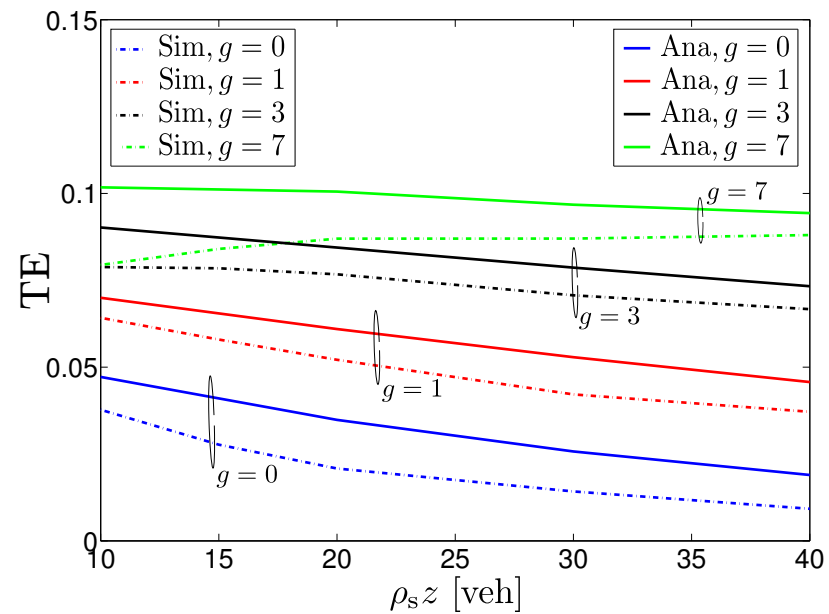

(c)

Figure 6 Simulation and analytical performance results of the polynomial protocol. (a) $D$, (b) $R E$, and (c) TE, as a function of $\rho_{s} z$, obtained using the polynomial protocol and different values of $\mathrm{g}$. The values $C W=31, I_{\text {norm }}=8, P=1000$ bytes, and $R=1 \mathrm{Mbps}$ are considered. Both simulation (Sim) and analytical results (Ana) are shown.

while for increasing values of $\rho_{\mathrm{s}} z$ (e.g., larger than 20 veh) its impact vanishes.

From the results in Figures 6 and 7, it emerges clearly that there is no optimal value of $g$. However, the proposed framework allows to optimize a single performance metric, after having imposed some constraints on the other metrics, on the basis of proper quality of service criteria. A possible choice consists in ignoring $\mathrm{TE}$ and minimizing $\mathrm{D}$ under the constraint of attaining a target value of RE. Since D is a decreasing function of $g$, it is possible to define the following quasi-optimal $g^{*}$ :

$$
g *\left(\rho_{\mathrm{s}} z\right)=\left\{\max (g) \mid R E\left(\rho_{\mathrm{s}} z\right)>0.95\right\} .
$$

Selecting $g=g^{*}$ allows to achieve the minimum delay under a constraint on the RE. The obtained $g^{*}$ is shown, as a function of $\rho_{\mathrm{s}} z$, in Figure 8a, and the following considerations can be drawn: (i) $g^{*}$ is an increasing monotonic function of $\rho_{\mathrm{s}} z$; (ii) with the exception of the region in proximity to $\rho_{\mathrm{s}} z=0$, where $g^{*}$ tends to $0, g^{*}$ has a quasi-linear dependence with respect to $\rho_{\mathrm{s}} z$. It can be shown that if $\mathrm{RE} \simeq 1$ for each value of $\rho_{\mathrm{s}} z$. Note that the selection of $g^{*}$ allows to maximize RE. However, as shown in Figure 8, D is always higher than $0.08 \mathrm{~s}$, a delay which is instead guaranteed by the use of $g=7$, as shown in the same figure.

\subsection{Silencing irresponsible forwarding}

As pointed out in Section 6, the proposed framework can be applied to a large family of broadcast protocols. In this section, the framework is applied to SIF. In particular, the validity of the proposed analytical framework is clearly shown in Figure 9, where (a) D, (b) RE, and (c) TE are shown, as functions of $\rho_{s} z$, for different values of $c$, by directly comparing both analytical and simulation results. As with the polynomial broadcast protocol, in 


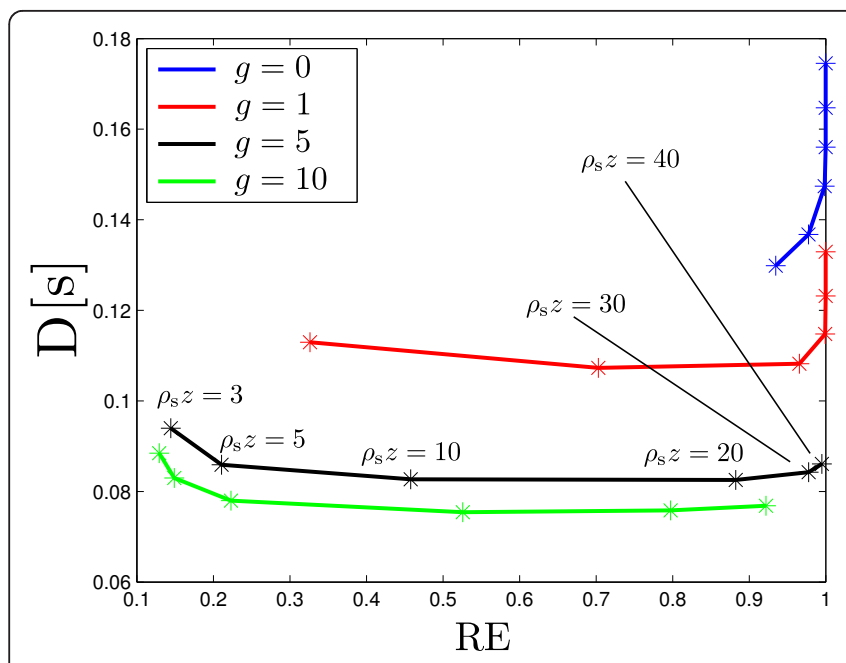

(a)

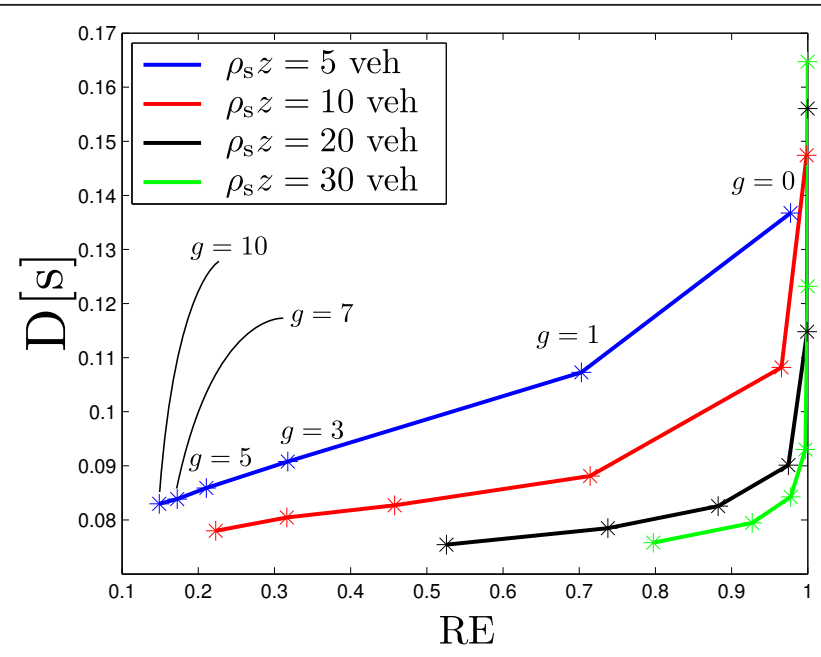

(b)

Figure 7 Analytical performance results of the polynomial protocol. $D$ as a function of RE, parametrized with respect to (a) $\rho_{\mathrm{s}} z$ (for various values of $g$ ) and (b) $g$ (for various values of $\rho_{s} z$ ). The results are obtained by considering $C W=31, I_{\text {norm }}=8, P=1000$ bytes, and $R=1$ Mbps.

this case as well there is a good agreement between the results obtained with the analytical model and the simulations. In particular, it can be observed that the accuracy of the model depends on the value of the shape parameter $c$ (the highest average accuracy, over all metrics, is observed with $c=7$ ). By comparing Figures 6 and 9, one can observe that polynomial and SIF protocols have a different dependence on $\rho_{s} z$. In particular, in the case of SIF, as the product $\rho_{s} z$ increases RE remains roughly the same, while D decreases and TE increases.
In other words, SIF performs better in dense networks. On the other hand, in the case of the polynomial protocol (Figure 6), D and TE have an opposite behavior (namely, D slightly increases and TE slightly decreases for increasing values of $\rho_{s} z$ ), and RE strongly depends on $\rho_{s} z$, especially in sparse networks. In general, SIF outperforms the polynomial broadcast protocol.

Furthermore, from Figure 9 it is clear that also for SIF there is no optimal value of the parameter $c$ which simultaneously optimizes the performance according to

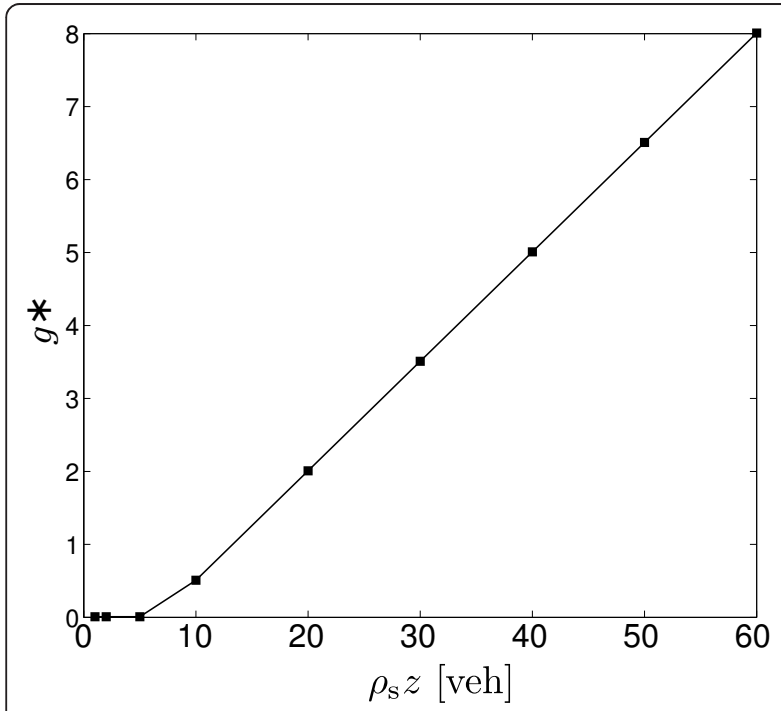

(a)

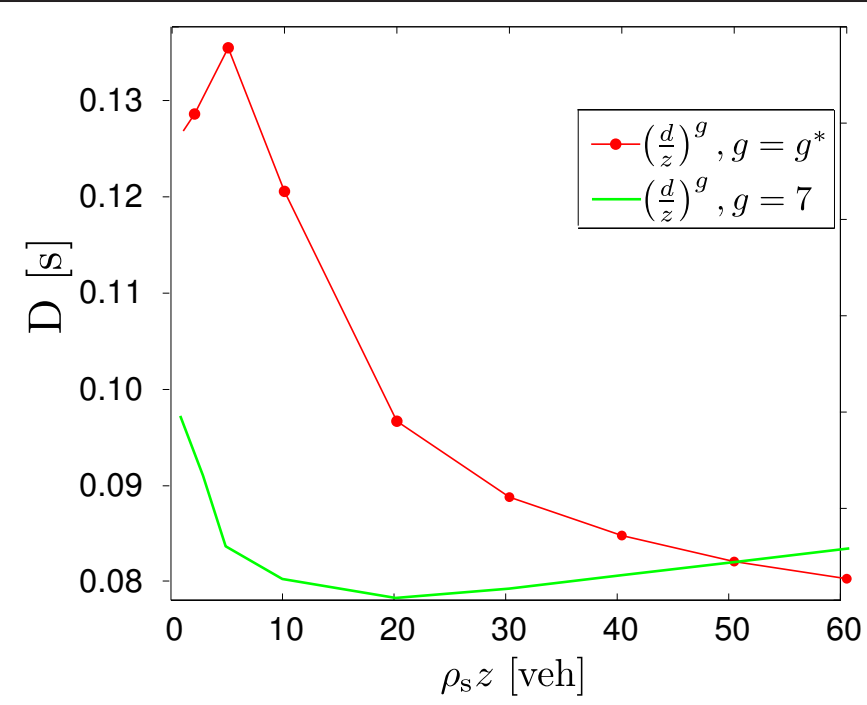

(b)

Figure 8 Optimal $g$ values for the polynomial protocol. (a) $g^{*}$ and (b) D, as a function of $\rho_{\mathrm{s}} z$ 


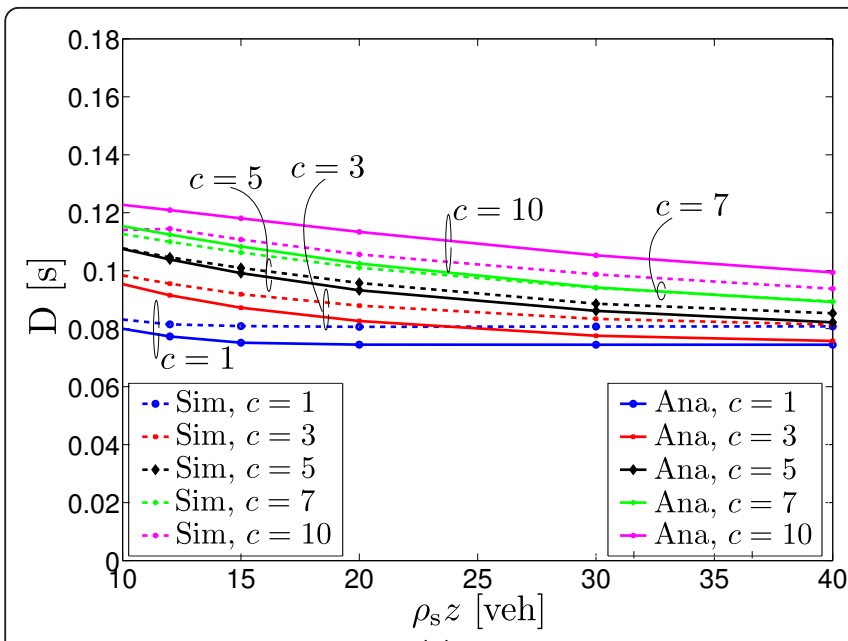

(a)

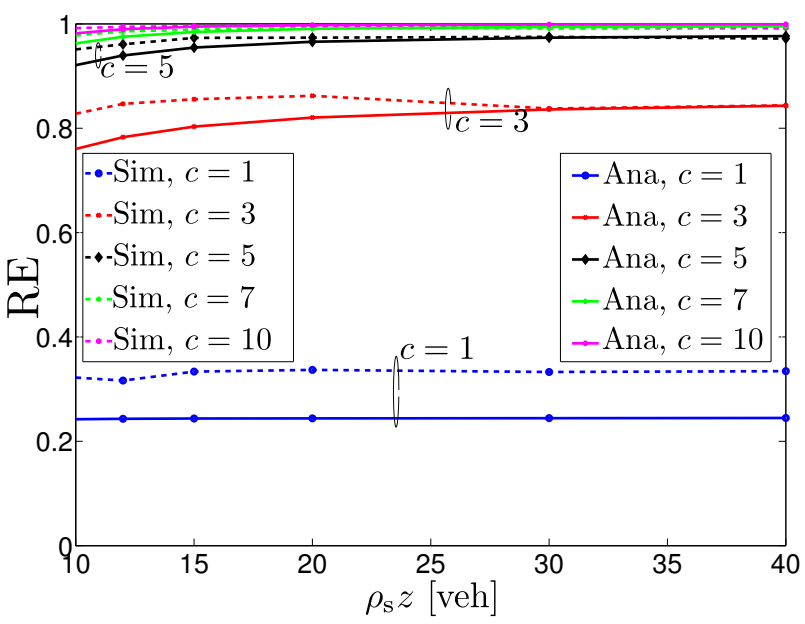

(b)

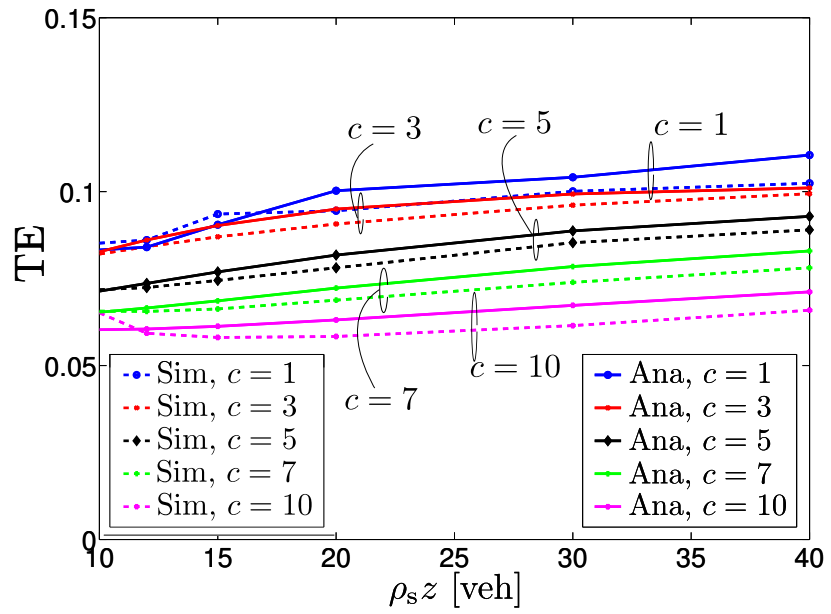

(c)

Figure 9 Analytical performance results of the SIF protocol. D as a function of RE, parametrized with respect to $\rho_{\mathrm{s}} Z$ (for various values of $c$ ) (a) and $c$ (for various values of $\rho_{\mathrm{s}} \mathrm{z}$ ) (b). The results are obtained by using the SIF protocol and considering $\mathrm{cW}=31, I_{\text {norm }}=8, P=1000$ bytes, and $R=1 \mathrm{Mbps}$.

all considered metrics. This fact can be better understood from Figure 10, where D is shown as a function of RE, parametrized, respectively, with respect to (a) $\rho_{s} z$ and (b) $c$. In particular, from Figure 10b it emerges that if one wants to guarantee a minimum value of RE (say 0.95 ), it is necessary to use a sufficiently high value of $c$. This, in turns, does not minimize $D$, which, as shown in Figure 10b, is directly proportional to $c$. Moreover, the results in Figure 10a strengthen the observations carried out regarding the results in Figure 9. In fact, they clearly evidence two important characteristics of SIF: (i) RE is not affected by the value of $\rho_{s} z$, as SIF automatically adapts; (ii) counterintuitively D is a decreasing function of $\rho_{s} z$ (e.g., SIF performs better in dense networks).

\subsection{Comparison with benchmark protocols}

As aforementioned, the theoretical framework presented in this manuscript can be used for evaluating a large number of broadcast protocols. In this subsection, it is applied to two benchmark broadcast protocols: (i) the flooding protocol (denoted with "FLOOD"), where each node forwards a received message; (ii) the optimal MCDS-based protocol (denoted with "MCDS"), where a hypothetical network genius selects as relays only the nodes belonging to the MCDS set (as described in Section 1). In both cases, the silencing mechanism is employed.

These benchmark protocols are compared with the SIF and polynomial protocols, considering a vehicle spatial distribution characterized by a Poisson distribution with parameter $\rho_{s} z=16 \mathrm{veh}$. In order to have a significant comparison, the optimal values of $c$ and $g\left(c^{*}=4.8\right.$ and $\left.g^{*}=2.7\right)$ are considered. These values, obtained through the analytical framework, allow to minimize D under the constraint of having a RE higher than 0.95 , in a scenario with $\rho_{\mathrm{s}} z=16 \mathrm{veh}$. The results, attained 


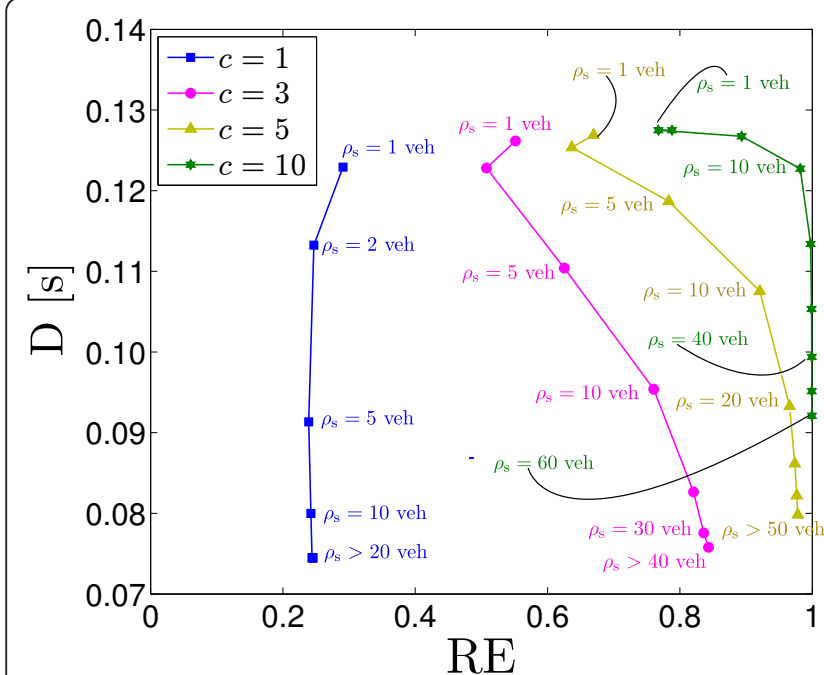

(a)

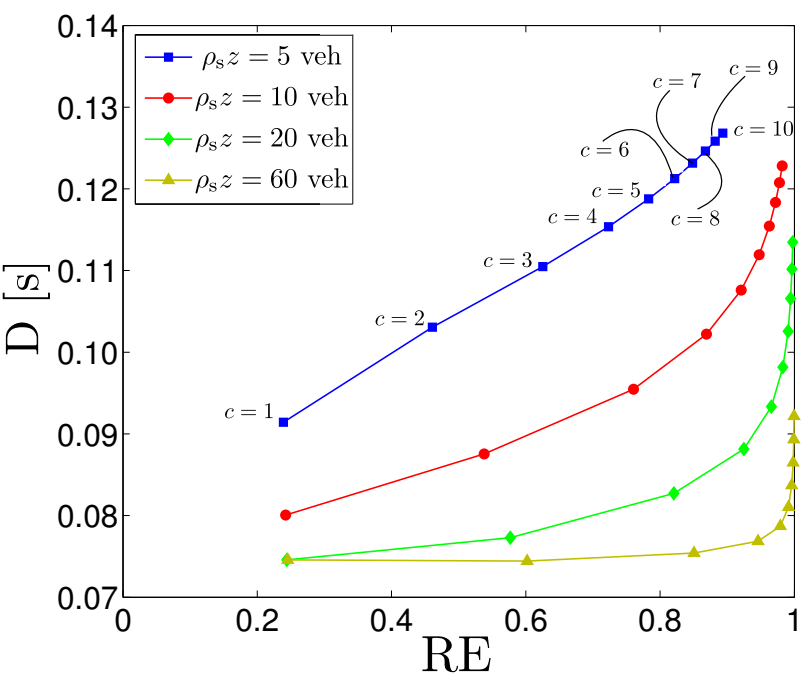

(b)

Figure 10 Simulation and analytical performance results of the SIF protocol. (a) D, (b) RE, and (c) TE, as a function of $\rho_{s} z$, obtained using the SIF protocol and different values of $c$. The values $C W=31, I_{\text {norm }}=8, P=1000$ bytes, and $R=1 \mathrm{Mbps}$ are considered. Both simulation (Sim) and analytical results (Ana) are shown.

through both simulations and theoretical analysis, are shown in Figure 11. From the results in Figure 11, a few considerations can be drawn. First, for all considered metrics, there is a performance loss between the MCDS-based and the optimized SIF/polynomial protocols. At the same time, the SIF/polynomial protocols exhibit a similar performance gain with respect to flooding (with the exception of the RE metric). It is also possible to observe that, counterintuitively, the SIF and the polynomial protocols offer a similar performance level. This result can be motivated by considering that their PAFs tend to converge to a common shape, when using, respectively, the optimal values $g^{*}$ and $c^{*}$ as their key parameters. Finally, it can be also be noticed an excellent match between simulation and theoretical results can be observed.

\subsection{Impact of topology on the protocol performance}

The goal of this subsection is to assess (a-posteriori) the validity of the assumption, made in Section 2, of considering a uni-dimensional static network. The validation is performed through simulations, by taking into account the protocols considered in Section 7.3 (namely, flooding, MCDS-based, SIF, and polynomial protocols). According to our assumption, we expect that the performances offered by these protocols will not be significantly affected by the network topology. To this end, we consider three different scenarios: (i) the uni-dimensional (single-lane) static network presented in Section 2; (ii) a multi-lane static network; (iii) a multi-lane mobile network. The multi-lane static scenario is composed by
$N_{\text {lane }}=6$ adjacent lanes, each with width equal to $w_{\text {lane }}=$ $4 \mathrm{~m}$. This network is obtained by simply replicating the single-lane topology. In particular, in each lane the positions of the vehicles are generated according to a PPP of parameter $\rho_{\mathrm{s}} / N_{\text {lane. }}$. Similarly, the multi-lane mobile scenario is composed by $N_{\text {lane }}=6$ adjacent lanes ( 3 per direction of movement), each with width equal to $w_{\text {lane }}=$ $4 \mathrm{~m}$. In this case, the vehicles are moving according to the intelligent driver motion with lane changes (IDMLC) mobility model [24] and, therefore, their positions do not have Poisson distribution. The mobility traces have been obtained using VanetMobiSim [25] and plugged in the ns-2 network simulator. The vehicles' speeds are independent and uniformly distributed in the interval $(20-40) \mathrm{m} / \mathrm{s}$. Greater insights about the mobility models and the trace generation process are provided in [26]. It should be noticed that the value of the per-lane vehicular density $\left(\rho_{\mathrm{s}}\right)$ is time-averaged, since it is computed directly from the mobility trace and thus is time-varying. In Figure 12, we show the results obtained by considering $\rho_{\mathrm{s}}=$ 16 veh and the optimal values of $c$ and $g\left(c^{*}=4.8\right.$ and $g^{*}$ $=2.7$ ). It can be easily noticed that the performances obtained in the considered scenarios are quite similar. Hence, this proves (a-posteriori) that the assumptions made in Section 2 are substantially correct. More specifically, it can be observed that increasing the width of the network leads to very similar values of $R E$ and $D$, and to slightly higher TE (this can be justified by considering that there is a higher number of nodes in the neighborhood of a vehicle). Instead, if we consider the same scenario but with mobile vehicles, one can observe that the 


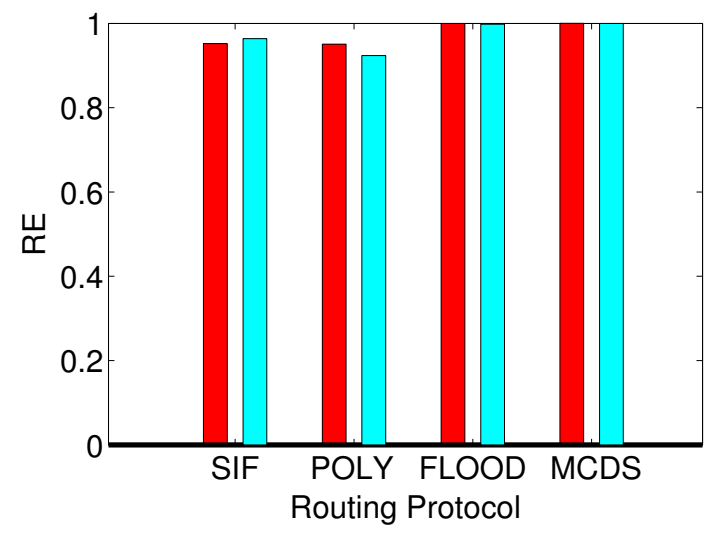

(a)

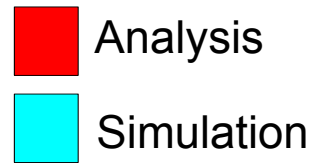

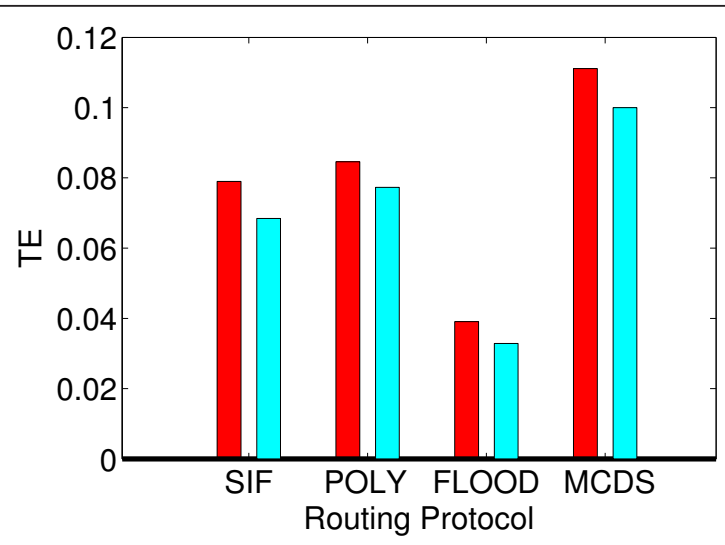

(b)

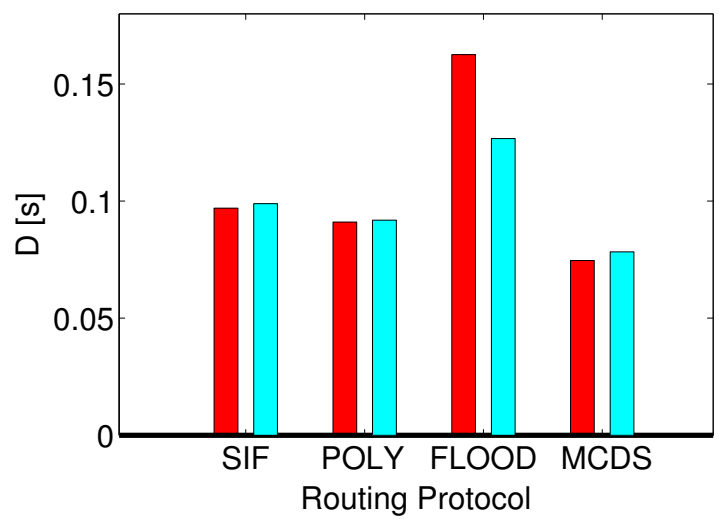

(c)

Figure 11 Simulation and analytical performance results of several protocols. (a) D, (b) RE, and (c) TE, obtained using the SIF, polynomial, flooding, and MCDS protocols, with $\rho_{s} z=16$ veh, $c^{*}=4.8$, and $g^{*}=2.7$. Both simulation and analytical results are shown.

RE becomes slightly lower, while D and TE become higher. This behavior is motivated by the tendency of mobile VANETs to form ephemeral clusters of vehicles [27], leading to a reduced RE and increased D but to a higher TE.

Finally, the limited impact of the vehicle mobility on the protocols' performance could have been expected by considering the values of the worst case transmission time (about $0.2 \mathrm{~s}$ ) and of the the maximum allowed speed (roughly equal to $40 \mathrm{~m} / \mathrm{s}$, corresponding to $144 \mathrm{~km} / \mathrm{h}$ ). In these conditions, two vehicles proceeding in opposite directions on a highway have a differential speed of $80 \mathrm{~m} / \mathrm{s}$, and this leads, in turn, to a distance variation of $16 \mathrm{~m}$ during a packet transmission time. A distance of $16 \mathrm{~m}$ (the worst-case variation) corresponds to a small fraction of the transmission range of a typical IEEE 802.11 network interface (in Figure 12, we have considered $z=160 \mathrm{~m}$ ).

\section{Conclusions}

In this article, we have presented a theoretical framework, based on a recursive computational approach, for average performance analysis of multihop broadcast protocols with silencing. We have then considered its application to VANET scenarios. The framework can be used in all the scenarios where the nodes' positions are distributed in such a way that their average positions are equally spaced. For example, it can be readily used for topologies where the nodes' positions have approximately a Poisson distribution. The proposed framework can be applied to a broad family of protocols and its validity has been assessed by means of ns2 simulations, by considering several VANET scenarios. In particular, the framework allows to characterize the average performance of broadcast multihop protocols in highway-like scenarios, either static or mobile, thus preventing the use of time-wasting numerical simulations.

\section{Appendix 1: Derivation of the average nodes positions}

In this appendix, we derive the average value of the positions vector $\mathbf{R}\left({ }^{n}\right)(n \in \mathbb{N})$ of $n$ Poisson points falling in the finite interval $\mathcal{I}=(0, z)$. The average values 


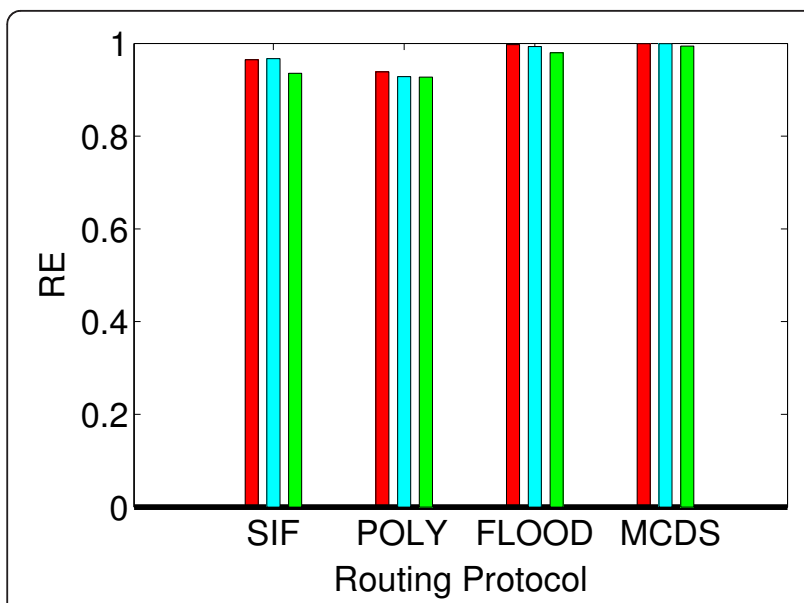

(a)

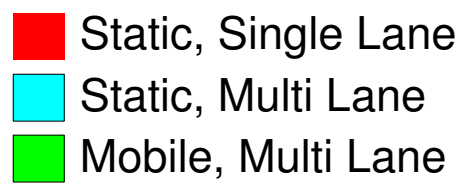

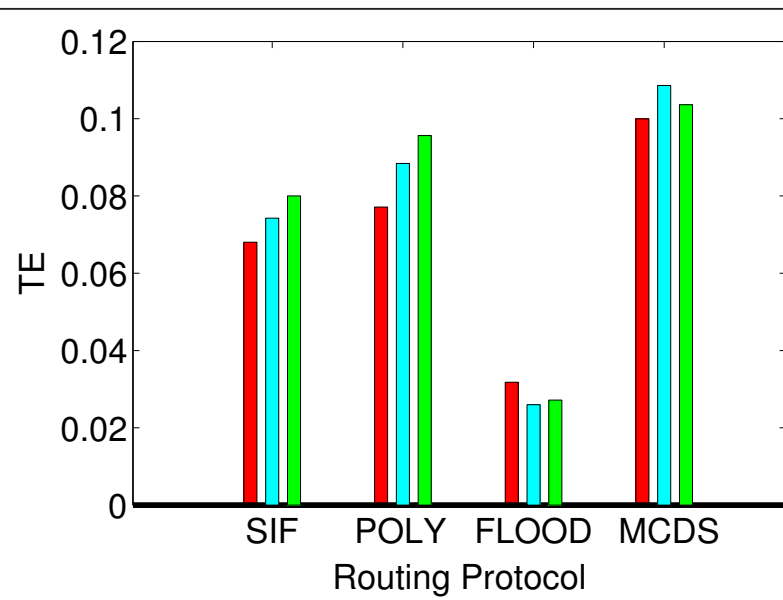

(b)

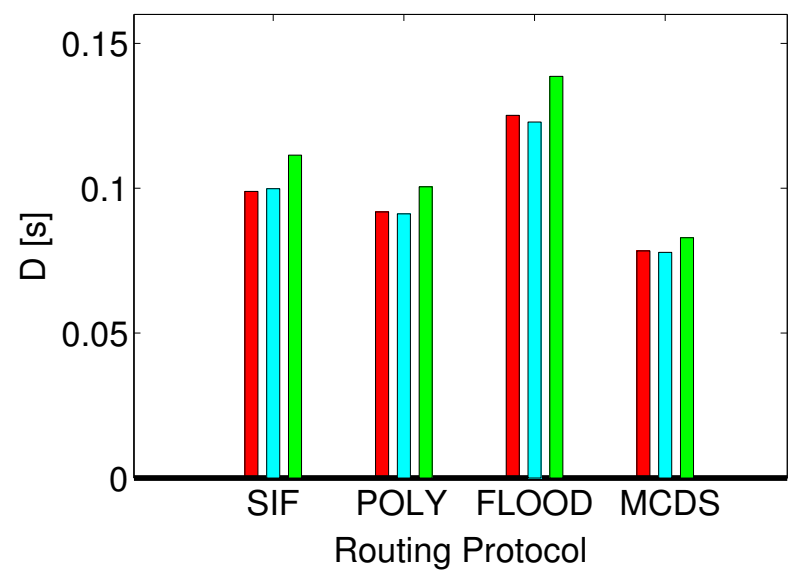

(c)

Figure 12 Simulation analysis of the impact of the network topology on the performance of several protocols. (a) $D$, (b) $R E$, and (c) TE, obtained using the SIF, polynomial, flooding, and MCDS protocols, with $\rho_{s} z=16$ veh, $c^{*}=4.8$, and $g^{*}=2.7$. The results are obtained through simulations by considering different topologies, namely: a single-lane static network, a multi-lane static network, and a multi-lane mobile network (highway-like).

can be computed by firstly deriving the joint PDF of the vector $\mathbf{R}^{(n)}$, denoted as $f_{\mathbf{R}}^{(n)}(\mathbf{r})$, and defined over a proper $n$-dimensional domain $\mathcal{D}_{n}$. From $f_{\mathbf{R}}^{(n)}(\mathbf{r})$, it is then possible to derive the marginal PDF of $R_{j}(j=1$, $2, \ldots, n)$, denoted as $f_{R_{j}}^{(n)}\left(r_{j}\right)$ and, from this, the average value $\bar{R}_{j}^{(n)}$.

\section{A single point in $\mathcal{I}$}

In this case, $n=1$ and $\mathcal{D}_{n}=\mathcal{I}$. In this case, $R_{1}$ has a uniform distribution in $\mathcal{I}$ and its (marginal) PDF is given by:

$$
f_{R_{1}}^{(1)}\left(r_{1}\right)=\left\{\begin{array}{l}
\frac{1}{z} r_{1} \in \mathcal{D}_{1} \\
0 \text { otherwise } .
\end{array}\right.
$$

The average value is:

$$
\bar{R}_{1}^{(1)}=\frac{z}{2} \text {. }
$$

\section{Two points in $\mathcal{I}$}

Without loss of generality, it is possible to order the points by imposing that $r_{2}>r_{1}$. Thanks to this assumption, $\mathcal{D}_{2}$ can be expressed as follows:

$$
\mathcal{D}_{2}=\left\{\left(r_{1}, r_{2}\right) \in \mathbb{R}^{2}: r_{1} \in(0, z), r_{2} \in(0, z), r_{1}<r_{2}\right\} .
$$

The joint PDF is uniform over $\mathcal{D}_{2}$ and can be expressed as follows:

$$
f_{R_{1} R_{2}}\left(r_{1}, r_{2}\right)=\left\{\begin{array}{ll}
\frac{1}{\operatorname{Area}\left(\mathcal{D}_{2}\right)} & \left(r_{1}, r_{2}\right) \in \mathcal{D}_{2} \\
0 & \text { otherwise }
\end{array}= \begin{cases}\frac{2}{z^{2}}\left(r_{1}, r_{2}\right) \in \mathcal{D}_{2} \\
0 & \text { otherwise }\end{cases}\right.
$$


From the joint PDF, the marginal PDFs of $R_{1}$ and $R_{2}$ can be obtained:

$$
\begin{aligned}
& f_{R_{1}}^{(2)}\left(r_{1}\right)=\int_{0}^{\infty} f_{R_{1} R_{2}}\left(r_{1}, r_{2}\right) \mathrm{d} r_{2}=\left\{\begin{array}{ll}
\int_{r_{1}}^{z} \frac{2}{z^{2}} \mathrm{~d} r_{2} & r_{1} \in(0, z) \\
0 & \text { otherwise }
\end{array}=\left\{\begin{array}{l}
\frac{2\left(z-r_{1}\right)}{z^{2}} r_{1} \in(0, z) \\
\text { otherwise }
\end{array}\right.\right. \\
& f_{R_{2}}^{(2)}\left(r_{2}\right)=\int_{0}^{\infty} f_{R_{1} R_{2}}\left(r_{1}, r_{2}\right) \mathrm{d} r_{1}=\left\{\begin{array}{ll}
\int_{0}^{r_{2}} \frac{2}{z^{2}} \mathrm{~d} r_{1} & r_{2} \in(0, z) \\
0 & \text { otherwise }
\end{array}= \begin{cases}\frac{2\left(z-r_{2}\right)}{z^{2}} & r_{2} \in(0, z) \\
0 & \text { otherwise. }\end{cases} \right.
\end{aligned}
$$

Using Equations (17) and (18), the average values of $R_{1}$ and $R_{2}$ can be expressed:

$$
\begin{aligned}
& \bar{R}_{1}^{(2)}=\int_{0}^{z} r_{1} \frac{2\left(z-r_{1}\right)}{z^{2}} \mathrm{~d} r_{1}=\frac{z}{3} \\
& \bar{R}_{2}^{(2)}=\int_{0}^{z} r_{2} \frac{2\left(z-r_{2}\right)}{z^{2}} \mathrm{~d} r_{2}=\frac{2}{3} z .
\end{aligned}
$$

\section{A generic number of $n$ points in $\mathcal{I}$}

As in the case with $n=2$, it is possible to order the points as that $r_{1}<r_{2}<\cdots<r_{n}$, without losing any generality. Hence, the $n$-dimensional domain can be expressed as follows:

$$
\mathcal{D}_{n}=\left\{\left(r_{1}, \ldots, r_{n}\right) \in \mathbb{R}^{n}: r_{i} \in(0, z) \forall i \in\{1, \ldots, n\}, r_{1}<r_{2}<\cdots<r_{n}\right\} .
$$

The joint PDF of the $n$ Poisson points has the following expression:

$$
f_{R_{1} \ldots R_{n}}\left(r_{1}, \ldots, r_{n}\right)=\left\{\begin{array}{ll}
\frac{1}{\operatorname{Volume}\left(\mathcal{D}_{n}\right)}\left(r_{1}, \ldots, r_{n}\right) \in \mathcal{D}_{n} \\
0 & \text { otherwise }
\end{array}= \begin{cases}\frac{n !}{z^{n}} & \left(r_{1}, \ldots, r_{n}\right) \in \mathcal{D}_{n} \\
0 & \text { otherwise }\end{cases}\right.
$$

where

$$
\begin{aligned}
& \operatorname{Volume}\left(\mathcal{D}_{n}\right)=\int_{0}^{z} \int_{r_{1}}^{z} \ldots \int_{r_{n-1}}^{z} 1 \mathrm{~d} r_{n} \cdots \mathrm{d} r_{2} \mathrm{~d} r_{1} \\
&=\int_{0}^{z} \int_{r_{1}}^{z} \ldots \underbrace{z}_{r_{n-2}}\left(z-r_{n-1}\right) \mathrm{d} r_{n-1} \cdots \mathrm{d} r_{2} \mathrm{~d} r_{1} \\
& \frac{\left(z-r_{n-2}\right)^{2}}{2} \\
& \vdots \\
&=\frac{z^{n}}{n \cdot(n-1) \cdot(n-2) \cdots 3 \cdot 2} \\
&=\frac{z^{n}}{n !} .
\end{aligned}
$$

The marginal PDF of the position of the $i$-th point is given by:

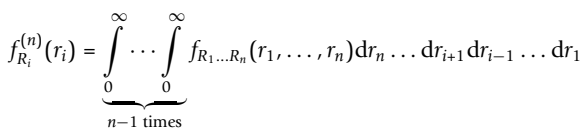

$$
\begin{aligned}
& =\int_{0}^{r_{i}} \int_{r_{1}}^{r_{i}} \ldots \int_{r_{i-2}}^{r_{i}} \int_{r_{i}}^{z} \ldots \int_{r_{n-1}}^{z} \frac{n !}{z^{n}} \mathrm{~d} r_{n} \ldots \mathrm{d} r_{i+1} \mathrm{~d} r_{i-1} \ldots \mathrm{d} r_{1} \\
& =\frac{n !}{z^{n}} \int_{0}^{r_{i}} \int_{r_{1}}^{r_{i}} \ldots \int_{r_{i-2}}^{r_{i}} \int_{r_{i}}^{z} \ldots \underbrace{z}_{\frac{\left(z-r_{n-2}\right)^{2}}{2}} \underbrace{\int_{r_{i}}}_{r_{n-2}}\left(z-r_{n-1}\right) \mathrm{d} r_{n-1} \ldots \mathrm{d} r_{i+1} \mathrm{~d} r_{i-1} \ldots \mathrm{d} r_{1} \\
& =\frac{n !}{z^{n}} \int_{0}^{r_{i}} \int_{r_{1}}^{r_{i}} \ldots \int_{r_{i-2}}^{r_{i}} \frac{\left(z-r_{i}\right)^{n-i}}{(n-i) \cdot(n-i-1) \ldots 3.2} \mathrm{~d} r_{i-1} \ldots \mathrm{d} r_{1} \\
& =\frac{n !}{z^{n}} \frac{\left(z-r_{i}\right)^{n-i}}{(n-i) !} \int_{0}^{r_{i}} \int_{r_{1}}^{r_{i}} \ldots \int_{r_{i-2}}^{r_{i}} \mathrm{~d} r_{i-1} \ldots \mathrm{d} r_{1} \\
& =\frac{n !}{z^{n}} \frac{\left(z-r_{i}\right)^{n-i}}{(n-i) !} \int_{0}^{r_{i}} \int_{r_{1}}^{r_{i}} \ldots \underbrace{\int_{r_{i-3}}^{r_{i}}\left(r_{i}-r_{i-2}\right)}_{\frac{\left(r_{i}-r_{i-3}\right)^{2}}{2}} \mathrm{~d} r_{i-2} \ldots \mathrm{d} r_{1} \\
& =\frac{n !}{z^{n}} \frac{\left(z-r_{i}\right)^{n-i}}{(n-i) !} \underbrace{\int_{0}^{r_{i}} \frac{\left(r_{i}-r_{1}\right)^{i-2}}{(i-2) \ldots 3.2}}_{\frac{r_{i}^{i-1}}{(i-1) \ldots . .3 .2}} \mathrm{~d} r_{1} \\
& =\frac{n !}{z^{n}} \frac{\left(z-r_{i}\right)^{n-i} r_{i}^{i-1}}{(n-i) !(i-1) !} \quad i=1, \ldots, n .
\end{aligned}
$$

On the basis of Equation (19), it is straightforward to derive the marginal PDF of $R_{i}(i=1,2, \ldots, n)$, given the presence of $n$ points in the interval $\mathcal{I}$ :

$$
f_{R_{i}}^{(n)}\left(r_{i}\right)= \begin{cases}\frac{n !}{z^{n}} \frac{\left(z-r_{i}\right)^{(n-i)} r_{i}^{i-1}}{(n-1) !(i-1) !} r_{i} \in(0, z) \quad i=1, \ldots, n \\ 0 \quad \text { otherwise. }\end{cases}
$$

Finally, from Equation (20) the average value of $R_{i}$ can be expressed as follows:

$$
\bar{R}_{i}^{(n)}=\int_{0}^{z} r_{i} \frac{n !}{z^{n}} \frac{\left(z-r_{i}\right)^{n-i} r_{i}^{i-1}}{(n-i) !(i-1) !} \mathrm{d} r_{i}=i \frac{z}{n+1} \quad i=1, \ldots, n .
$$

\section{Appendix 2: Per-node retransmission probability in a network with equally spaced nodes}

We consider the deterministic scenario introduced in Section 6.1, composed by a fixed number $n$ of nodes equally spaced in the interval $\mathcal{I}=(0, z) \subset \mathbb{R}$, with the positions vector $\mathbf{R}\left({ }^{n}\right)$ defined in Equation (1). In this appendix, we derive the following probabilities:

$$
p_{\mathrm{rtx}}^{(n)}(i)=\mathrm{P}\left\{S_{i}\right\} \quad i=1,2, \ldots, n
$$


where the event $\mathcal{S}_{i}$ was defined in Section 6.1. In order to derive $p_{\mathrm{rtx}}^{(n)}(i)$, it is helpful to introduce the following auxiliary events:

- $\mathcal{B}_{i} \triangleq$ the node $i$ is designated as a relay\};

- $\mathcal{C}_{i} \triangleq$ the node $i$ wins the contention among a set of $n$ nodes $\}$;

- $\mathcal{D}_{i}^{(m)} \triangleq \mathrm{A}$ \{the node $i$ wins the contention among a set of $m$ contending nodes $\}$;

- $\mathcal{W}_{k} \triangleq$ the value $\mathrm{BC}=k$ is chosen by a single node $k=0, \ldots, c w-1$;

- $\mathcal{W} \triangleq\{$ at least a value of $\mathrm{BC} \in[0, c w-1]$ is chosen by a single node\}.

The event $\mathcal{S}_{i}$, defined in Subsection 6.1, is verified if both the events $\mathcal{B}_{i}$ and $\mathcal{C}_{i}$ happen. Therefore, $p_{\mathrm{rtx}}^{(n)}(i)$ can be expressed as:

$$
p_{\text {rtx }}^{(n)}(i)=\operatorname{P}\left\{\mathcal{S}_{i}\right\}=\operatorname{P}\left\{\mathcal{B}_{i} \cap \mathcal{C}_{i}\right\}=\operatorname{P}\left\{\mathcal{B}_{i}\right\} \operatorname{P}\left\{\mathcal{C}_{i}\right\}
$$

where the last equality is motivated by the independence of the events $\mathcal{B}_{i}$ and $\mathcal{C}_{i}$. The probability $\mathrm{P}\left\{\mathcal{B}_{i}\right\}$ is known, since it should be replaced with one of the PAF used by the protocols considered in this work (defined in Equations (4) and (5)). On the opposite, the unknown probability $\mathrm{P}\left\{\mathcal{C}_{i}\right\}$ can be derived by applying the total probability theorem, thus obtaining:

$$
\begin{aligned}
p_{\mathrm{rtx}}^{(n)}(i) & =P\left\{\mathcal{B}_{i}\right\} \mathrm{P}\left\{\mathcal{C}_{i}\right\} \\
& =p_{i} \sum_{m=1}^{n} \mathrm{P}\left\{\mathcal{D}_{i}^{(m)}\right\} p_{V_{i}^{(n)}}(m-1) \\
& =p_{i} \sum_{m=1}^{n} q^{(m)}(i) p_{V_{i}^{(n)}}(m-1)
\end{aligned}
$$

where $q^{(m)}(i) \triangleq P\left\{\mathcal{D}_{i}^{(m)}\right\}$ and $V_{i}^{(n)} \in\{0, \ldots, n-1\}$ is a discrete random variable defined as:

$$
V_{i}^{(n)} \triangleq\{\text { the number of nodes competing with node } i \text { given } n \text { nodes }\} .
$$

It can be shown that the PMF of $V_{i}^{(n)}$ can be expressed as follows:

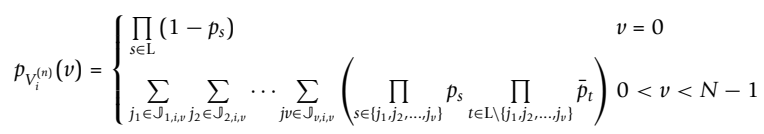

where $\mathbb{L} \triangleq\{1,2, \ldots, N\} \backslash\{i\}$ and the sets $\left\{\mathbb{J}_{k, i, v}\right\}$ are defined as follows:

$$
J_{k, i, v}= \begin{cases}\{k+1, k+2, \ldots, n-v+k\} & i \leq k \\ \{k, k+1, \ldots, i-1, i+1, \ldots, n-v+k\} & k<i \leq n-v+k-1 \\ \{k, k+1, \ldots, n-v+k-1\} & i>-v+k-1 .\end{cases}
$$

The probability $q^{(m)}(i)$ can be computed by analyzing the BA mechanism of the IEEE 802.11b standard. In particular, it emerges that $q^{(m)}(i)$ is independent of $i$ and can be expressed as follows:

$$
q^{(m)}(i)=q^{(m)}=\frac{\mathrm{P}\{\mathcal{W}\}}{n}=\frac{\mathrm{P}\left\{\bigcup_{k=0}^{c w-1} \mathcal{W}_{k}\right\}}{n} .
$$

Since the events $\left\{\mathcal{W}_{k}\right\}$ are not disjoint, it is necessary to use the generalized union probability formula [29, Ch.4] to compute $\mathrm{P}\{\mathcal{W}\}$ :

$$
\begin{aligned}
\mathrm{P}\{\mathcal{W}\}= & \mathrm{P}\left\{\bigcup_{k=0}^{c w-1} \mathcal{W}_{k}\right\}=\sum_{k_{1}=0}^{c w-1} \mathrm{P}\left\{\mathcal{W}_{k_{1}}\right\} \\
& -\sum_{k_{1}<k_{2}} \mathrm{P}\left\{\mathcal{W}_{k_{1}} \cap \mathcal{W}_{k_{2}}\right\} \\
& +\sum_{k_{1}<k_{2}<k_{3}} \mathrm{P}\left\{\mathcal{W}_{k_{1}} \cap \mathcal{W}_{k_{2}} \cap \mathcal{W}_{k_{3}}\right\} \\
& +\cdots+ \\
& (-1)^{c w+1} \mathrm{P}\left\{\mathcal{W}_{0} \cap \mathcal{W}_{1} \cap \cdots \cap \mathcal{W}_{c w-1}\right\} .
\end{aligned}
$$

Since the addenda of each single sum of the righthand side of (22) are the same, taking into account the number of possible combinations, the generic righthand side of (22) can be then expressed as follows:

$$
(-1)^{r+1} \sum_{k_{1}<k_{2}<\cdots<k_{r}} P\left\{\mathcal{W}_{k_{1}} \cap \mathcal{W}_{k_{2}} \cap \cdots \cap \mathcal{W}_{k_{k}}\right\}=(-1)^{r+1}\left(\begin{array}{c}
c w \\
r
\end{array}\right) \frac{(c w-r)^{n-r} \prod_{j=0}^{r-1}(n-j)}{(c w)^{n}} .
$$

Thanks to Equation (22), $q^{(n)}$ can be finally given the following expression:

$$
q^{(n)}=\frac{\sum_{r=1}^{\min (n, c w)}(-1)^{r+1}\left(\begin{array}{c}
c w \\
r
\end{array}\right)(c w-r)^{n-k} \prod_{j=0}^{r-1}(n-j)}{n(c w)^{n}}
$$

where the term $\min (n, c w)$ is introduced to deal with the case $n<c w$.

\section{Appendix 3: Per-node delay in a network with equally spaced nodes}

In this appendix, we derive the number of slots spent by the $i$-th node during the backoff conditioned to the event $\mathcal{S}_{i}$, denoted as $N_{i \mid i}^{\text {bo }}$. By analyzing the BA mechanism of the IEEE 802.11b standard, one obtains:

$$
\bar{N}_{i \mid i}^{\mathrm{bo}}=\mathbb{E}\left[N_{i \mid i}^{\mathrm{bo}}\right]=\sum_{v=0}^{N-1} \mathbb{E}\left[N_{i}^{\mathrm{bo}} \mid V_{i}^{(N)}=v, \mathcal{S}_{i}\right] P\left\{V_{i}^{(N)}=v \mid \mathcal{S}_{i}\right\}
$$

where $P\left\{V_{i}^{(N)}=v \mid \mathcal{S}_{i}\right\}$ can be derived by means of the Bayes theorem as follows:

$$
P\left\{V_{i}^{(N)}=v \mid \mathcal{S}_{i}\right\}=\frac{\overbrace{P\left\{\mathcal{S}_{i} \mid V_{i}^{(N)}=v\right\}}^{p_{i} q^{(v+1)}} \overbrace{P\left\{V_{i}^{(N)}=v\right\}}^{p_{v_{i}^{(N)}}^{(v)}}}{\underbrace{P\left\{\mathcal{S}_{i}\right\}}_{p_{\mathrm{rx}}^{(N)}(i)}}=\frac{p_{i} q^{(v+1)} p_{V_{i}^{(N)}}(v)}{p_{\mathrm{rx}}^{(N)}(i)} .
$$


Instead, $\mathbb{E}\left[N_{i}^{\text {bo }} \mid V_{i}^{(N)}=v, \mathcal{S}_{i}\right]$ can be derived by observing that the delay associated with the event (the node $i$ transmits with success given $v$ contending nodes $\}$ depends on two factors: (i) the slot $B C_{i} \in\{0, \ldots, c w-1\}$ selected by the node $i$ for transmitting; (ii) the number of collisions occurred in the slots $0, \ldots, k-1$, which, given that $B C_{i}=k$, corresponds to the following random variable:

$$
N_{k, v}^{\mathrm{col}}=\{\text { number of collisions in slots } 0, \ldots, k-1\} N_{k, v}^{\mathrm{col}} \in\left\{0, J_{k, v}\right\}
$$

where $J_{k, v} \triangleq \min (k,\lfloor(v / 2)\rfloor)$ denotes the maximum number of collisions that can happen in slots $0, \ldots, k-1$. On the basis of these considerations it can be shown that:

$$
\begin{aligned}
& \mathbb{E}\left[N_{i}^{\mathrm{bo}} \mid V_{i}^{(N)}=v, \mathcal{S}_{i}\right]=\sum_{k=0}^{c w-1} \sum_{j=0}^{J_{k, v}} \mathbb{E}\left[N_{\mathrm{i}}^{\mathrm{bo}} \mid\left\{V_{i}^{(N)}=v, \mathcal{S}_{i}, B C_{i}=k, N_{k, v}^{\mathrm{col}}=j\right\}\right] . \\
& \mathrm{P}\left\{B C_{i}=k \wedge N_{k, v}^{\mathrm{col}}=j \mid V_{i}^{N}=v S_{i}\right\} \\
&=\sum_{k=0}^{c w-1} \sum_{j=0}^{J_{k, v}}\left(k+j T^{\mathrm{tx}}\right) P_{v}(k, j) v=0, \ldots, N-1
\end{aligned}
$$

where $P_{v}(k, j) \triangleq \mathrm{P}\left\{B C_{i}=k \wedge N_{k, v}^{\mathrm{col}}=j \mid V_{i}^{(N)}=v, \mathcal{S}_{i}\right\}$ is the $(k, j)$-th element of the matrix $\mathbf{P}_{v}$, of dimension $c w$ $\times\left(J_{c w-1, v}+1\right)$. There exist $N$ matrices $\mathbf{P}_{v}$, one for each value of $\left\{V_{i}^{(N)}=v\right\}, v \in\{0, N-1\}$.

In order to derive $\mathbf{P}_{\mathrm{v}}$ it is necessary to define the following random variables:

$$
\begin{aligned}
& H_{k, v}=\{\text { number of nodes with } B C<k\} \quad H_{k, v} \in\{0, \ldots, v\} \\
& =\left\{\begin{array}{ll}
\sum_{m=1}^{v} I_{m, k} k>0 \wedge v>0 \\
0 \quad \text { otherwise }
\end{array} \quad I_{m, k}= \begin{cases}1 & B C_{m}<k \\
0 & B C_{m}\end{cases} \right. \\
& N_{k, v \mid h}^{\text {opp }}=\left\{\text { number of nodes with } B C=k \mid H_{k, v}=h\right\} N_{k, v \mid h}^{\text {opp }} \in\{0, \ldots, v-h\} \\
& =\left\{\begin{array}{cc}
\sum_{m=1}^{v-h} L_{m, k} k \geq 0 \wedge v>0 \\
0 \quad \text { otherwise }
\end{array} \quad L_{m, k}= \begin{cases}1 & B C_{m}=k \\
0 & B C_{m}>k .\end{cases} \right.
\end{aligned}
$$$$
N_{k, v) h}^{\text {col }}=\left\{\text { number of collisions in the } 0, \ldots, k-1 \mid H_{k, v}=h\right\} \quad N_{k, v, \mid h}^{\text {col }} \in\left\{0, \cdots, J_{k, h}\right\}
$$

It is then possible to compute $P_{v}(k, j)$ using Bayes theorem and the total probability theorem:

$$
\begin{aligned}
& P_{v}(k, j)=\mathrm{P}\left\{B C_{i}=k \wedge N_{k, v}^{\text {col }}=j \mid V_{i}^{(N)}=v, \mathcal{S}_{i}\right\}=\frac{\mathrm{P}\left\{\mathcal{S}_{i} \wedge B C_{i}=k \wedge N_{k, \nu}^{\text {col }}=j \mid V_{i}^{(N)}=v\right\}}{\mathrm{P}\left\{\mathcal{S}_{i} \mid V_{i}^{(N)}=v\right.} \\
& =\frac{P\left\{\mathcal{S}_{i} \wedge N_{k, v}^{\mathrm{col}}=j \mid V_{i}^{(N)}=v, B C_{i}=k,\right\} \mathrm{P}\left\{\mathrm{BC}_{i}=k \mid V_{i}^{(N)}=v\right\}}{p_{i} q^{(v+1)}} \\
& =\frac{1}{c w p_{i} q^{(v+1)}} \sum_{h=0}^{v} \mathrm{P}\left\{\mathcal{S}_{i} \wedge N_{k, v \mid h}^{c c l}=j \mid\left\{V_{i}^{(N)}=v, B C_{i}=k, H_{k, v}=h\right\}\right\} . \\
& P\left\{H_{k, v}=h \mid\left\{V_{i}^{(N)}=v, B C_{i}=k\right\}\right\} \\
& =\cdots \text {. } \\
& =\frac{1}{c w q^{(v+1)}} P^{\prime}{ }_{v}(k, j) \\
& =\frac{1}{c w q^{(v+1)}}\left\{\begin{array}{lrl}
\left(\frac{c w-1}{c w}\right)^{v} & k=0 \\
\sum_{h=0}^{v} M_{k, v}(j, h) N_{k, v}(0, h) & k=0, \ldots, c w-1
\end{array}\right. \\
& =\frac{1}{c w q^{(v+1)}} \quad\left(\frac{c w-1}{c w}\right)^{v} k=0
\end{aligned}
$$

where the $(j, h)$-th elements of matrix $\mathrm{N}_{k, v}$ (with dimension $(v+1) \times(v+1))$ are defined as:

$$
\begin{aligned}
N_{k, v}(n, h) & =P\left\{N_{k, v \mid h}^{\text {opp }}=n \mid\left\{V_{i}^{(N)}=v, B C_{i}=k, H_{k, v}=h\right\}\right\} \\
& =(v-h) \frac{(c w-k-1)^{v-h-n}}{(c w-k)^{v-h}} \\
v & =0, \ldots, N-1 \quad k=1, \ldots, c w-1 \quad h, n=0, \ldots, v
\end{aligned}
$$

while the $(j, h)$-th elements of matrix $\mathbf{M}_{k, v}$ (with dimension $\left.\left(J_{k, v}+1\right) \times(\mathrm{v}+1)\right)$ are defined as:

$$
\begin{aligned}
M_{k, v}(j, h) & =P\left\{N_{k, v \mid h}^{\mathrm{win}}=0 \wedge N_{k, v \mid h}^{\mathrm{col}}=j \mid\left\{V_{i}^{(N)}=v, B C_{i}=k, H_{k, v}=h\right\}\right\} . \\
& P\left\{H_{k, v}=h \mid\left\{V_{i}^{(N)}=v, B C_{i}=k\right\}\right\} \\
v & =0, \ldots, N-1 \quad k=1, \ldots, c w-1 \\
j & =0, \ldots, J_{k, v} \quad h=0, \ldots, v
\end{aligned}
$$

In order to reduce the computational burden, the matrix $\mathbf{M}_{k, v}$ can be derived by means of a recursive strategy. In particular, it can be observed that the number of collisions at the $k$-th hop is identical to (if nobody select the value $\mathrm{BC}=k-1$ ) or greater than 1 (if at least two nodes selects that value). Hence, once derived $\mathbf{M}_{1, v}$ it is possible to determine $\mathbf{M}_{k, v}$ for all the remaining values of $k$. In particular, the direct formulation for $k=1$ is the following:

$$
M_{1, v}(j, h)=\left\{\begin{array}{clrl}
\left(\frac{c w-k}{c w}\right) & j=0, & h=0 \\
0 & j=0, & h>0 \\
0 \quad j=1, & h \leq 1 \\
\left(\begin{array}{c}
v \\
h
\end{array}\right) \frac{(c w-k)^{v-h}}{(c w)^{v}} j=1, & 1<h<v
\end{array}\right.
$$

from which it is possible to derive $\mathbf{M}_{k+1, v}$ for any values of $k$ :

$$
M_{k+1, v}(j, h)=\left\{\begin{array}{l}
0 \quad h=1 \\
\left(\frac{c w-k+1}{c w}\right)^{v} j=0, \quad h=0 \\
I_{j, k} M_{k, \nu}(j, h) N_{k, \nu}(0, h)+\sum_{t=2 j-2}^{h-2} M_{k, \nu}(j-1, t) N_{k, \nu}(h-t, t) j \in \mathcal{J}_{k, \nu}, h \in\{2 j, v\} \\
0 \text { otherwise }
\end{array}\right.
$$

where the indicator function $I_{j, k}$ is defined as

$$
I_{j, k} \triangleq\left\{\begin{array}{l}
1 j \neq k \\
0 j=k
\end{array}\right.
$$

Finally, using Equations (24) and (25) in (23), one obtains the final expression:

$$
\begin{aligned}
\bar{N}_{i \mid i}^{\mathrm{bo}} & =\frac{p_{i}}{p_{\mathrm{rtx}}^{(N)}(i)} \sum_{v=0}^{N-1} q^{(v+1)} p_{V_{i}^{(N)}}(v) \sum_{k=0}^{c w-1} \sum_{j=0}^{J_{k, v}}\left(k+j T^{\mathrm{tx}}\right) P_{v}(k, j) \\
& =\frac{p_{i}}{c w p_{\mathrm{rxx}}^{(N)}(i)} \sum_{v=0}^{N-1} p_{V_{i}^{(N)}}(v) \sum_{k=0}^{c w-1} \sum_{j=0}^{J_{k, v}}\left(k+j T^{\mathrm{tx}}\right) P^{\prime}{ }_{v}(k, j) \\
& =\frac{p_{i}}{c w p_{\mathrm{rtx}}^{(N)}(i)} \sum_{v=0}^{N-1} p_{V_{i}(N)}(v) \sum_{k=1}^{c w-1}\left[k \sum_{j=0}^{J_{k, v}} P^{\prime}{ }_{v}(k, j)+T^{\mathrm{tx}} \sum_{j=1}^{J_{k, v}} j P^{\prime}{ }_{v}(k, j)\right]
\end{aligned}
$$


This allows to determine $D_{i \mid i}$ for every node of a given TD.

\section{Appendix 4: Recursive approach for the evaluation of the performance global metrics}

In this appendix, we outline the recursive approach which, coherently with an inductive principle, allows to derive the average global performance metrics (namely, $\mathrm{RE}, \mathrm{D}$, and TE), on the basis of the average local performance metrics of a generic TD. We recall that, thanks to the assumptions of the deterministic approach, all the TDs are identical and composed of $n$ nodes. The recursive approach is detailed by considering the computation of $\mathrm{D}$, but with the same approach it is also possible to derive RE and TE. The computation of the average $\mathrm{D}$ is carried out taking into account only the packets successfully arriving at the last reachable node, ignoring the unsuccessful retransmissions.

For all the values of $m$ such that $m \leq n$, all the $n$ nodes within the 1-st TD are reached by the source. Therefore, the average delay coincides with the average transmission time of the source, given by Equation (11), i.e.,

$$
\bar{D}^{(m)}=\bar{T}_{\mathrm{src},}^{\mathrm{tx}} \quad 1<m \leq n .
$$

However, for all the values $m>n$, at least a retransmission is necessary to reach the $m$-th node. In particular, if we consider the case $m=n+1$, the $(n+1)$-th node can be reached only and only if a successful transmission is carried out by a node of the 1-st TD. This event can happen in $n$ different ways, each associated with a different delay. The tree of the possible decisions is represented in Figure 13, where every branch is labeled with the associated probability and with the corresponding value of delay. Since we are conditioning to the fact of having a successful transmission, the probability of the event $\left\{\right.$ the $i$-th node transmits\} is given by $p_{Y}(i \mid \mathcal{S})$. Therefore, the average delay $\bar{D}^{(n+1)}$ can be obtained as follows:

$$
\bar{D}^{(n+1)}=T_{\mathrm{src}}^{\mathrm{tx}}+\sum_{i=1}^{n} \bar{D}_{i \mid i} p_{Y}(i \mid \mathcal{S}) .
$$

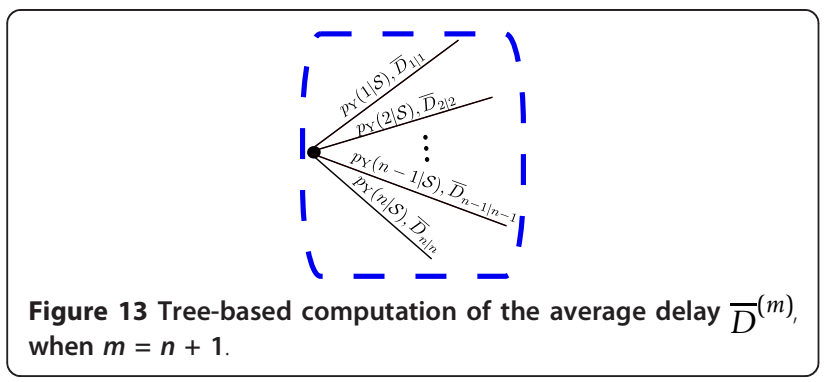

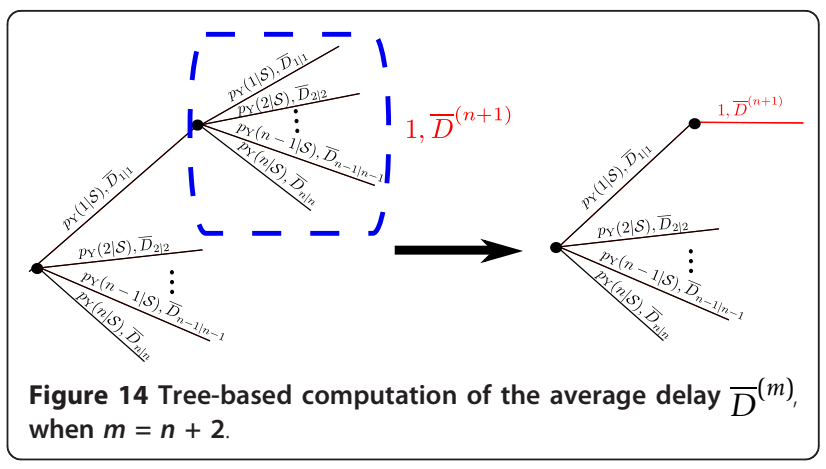

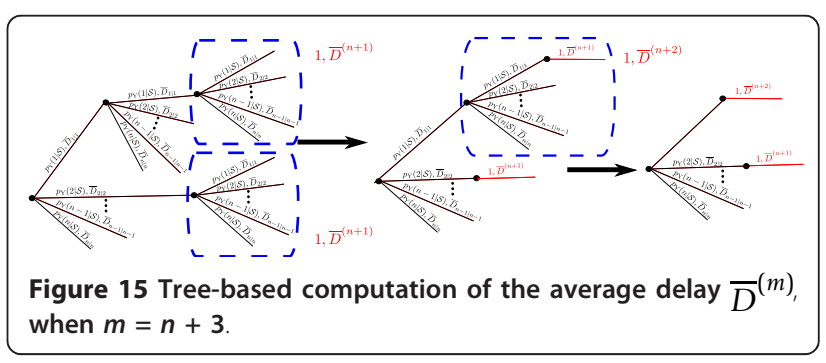

When $m=n+2$ the situation is slightly more complicated, since when the 1-st node is selected in the 1-st TD, two transmissions are needed to reach the $(n+2)-$ th node. In this case, a second TD, identical to the first, is formed, thus leading to the addition of $n$ branches to the tree, as shown in Figure 14. However, since the two TDs are identical, the branches following the event $\{y=1 \mid \mathcal{S}\}$, can be replaced by the average delay computed for $m=n+1$. Therefore, one obtains:

$$
\bar{D}^{(n+2)}=T_{\mathrm{src}}^{\mathrm{tx}}+\left(\bar{D}^{(n+1)}+\bar{D}_{i \mid i}\right) p_{Y}(1 \mid \mathcal{S})+\sum_{i=2}^{n} \bar{D}_{i \mid i} p_{Y}(i \mid \mathcal{S})
$$

Similar considerations can be drawn in the case with $m=n+3$ : the corresponding tree is shown in Figure 15. In this case, the two circled branches in the left figure, can be replaced by $\bar{D}^{(n+1)}$, obtaining the tree in the central figure that can be further simplifying by using $\bar{D}^{(n+2)}$, thus leading to the following expression:

$$
\bar{D}^{(n+3)}=T_{\mathrm{scc}}^{\mathrm{tx}}+\left(\bar{D}^{(n+2)}+\bar{D}_{1 \mid 1}\right) p_{\mathrm{Y}}(1 \mid \mathcal{S})+\left(\bar{D}^{(n+1)}+\bar{D}_{2 \mid 2}\right) p_{\mathrm{Y}}(2 \mid \mathcal{S})+\sum_{i=3}^{n} \bar{D}_{i|i| p_{Y}}(i \mid \mathcal{S}) .
$$

Now, by induction it is possible to derive the formulation of $\bar{D}^{(N)}$ given in (10).

\footnotetext{
Abbreviations

VANETs: vehicular ad-hoc NETworks; MANETs: mobile ad-hoc NETworks; MCDS: minimum connected dominant set; RTS/CTS: ready-to-send/clear-tosend; UMB: urban multihop broadcast; SB: smart broadcast; BPAB: binary partition assisted protocol; PPP: poisson point process; GPS: global positioning system; RE: REachability; TE: transmission efficiency; D: delay; MAC: medium access control; BA: basic access; DIFS: distributed interframe
} 
space; BC: backoff counter; PAF: probability assignment function; TD: transmission domain.

\section{Acknowledgements}

This work is carried out under the one-year project "Cross-Network Effective Traffic Alerts Dissemination" (X-NETAD, Eureka Label E! 6252 [28]), sponsored by the Ministry of Foreign Affairs (Italy) and The Israeli Industry Center for R\&D (Israel) under the "Israel-Italy Joint Innovation Program for Industrial, Scientific and Technological Cooperation in R\&D." The authors would like to thank Prof. A. Bononi of the University of Parma for his support and help.

\section{Competing interests}

The authors declare that they have no competing interests.

Received: 25 July 2011 Accepted: 12 January 2012

Published: 12 January 2012

\section{References}

1. S Ni, Y Tseng, Y Chen, J Sheu, The Broadcast Storm Problem in a Mobile Ad Hoc Network, in Proc. ACM Intern. Conf. on Mobile Comput. and Networking, (MOBICOM), Seattle, WA, USA, (1999), pp. 151-162

2. A Zanella, G Pierobon, S Merlin, On the limiting performance of broadcast algorithms over unidimensional ad-hoc radio networks, in Proc. of IEEE Intl. Conf. on Wireless Personal Multimedia Communications, (WMPC), Abano Terme, Italy Sept 2004, pp. 165-169. (2004)

3. M Torrent-Moreno, J Mittag, P Santi, H Hartenstein, Vehicle-to-vehicle communication: fair transmit power control for safety-critical information. IEEE Trans. Veh. Technol. 58(7), 3684-3707 (2009)

4. M Kihl, M Sichitiu, HP Joshi, Design and evaluation of two geocast protocols for vehicular ad-hoc networks. J Internet Eng. 2, 127-135 (2008)

5. G Korkmaz, E Ekici, F Özgüner, U Özgüner, Urban multi-hop broadcast protocol for inter-vehicle communication systems, in Proc. of ACM Intl. Workshop on Vehicular ad hoc networks, (VANET), (ACM, Philadelphia, USA, 2004), pp. 76-85

6. G Korkmaz, E Ekici, F Ozguner, Black-burst-based multihop broadcast protocols for vehicular networks. IEEE Trans. Veh. Technol. 56(5), 3159-3167 (2007)

7. J Sobrinho, A Krishnakumar, Quality-of-service in ad hoc carrier sense multiple access wireless networks. IEEE J Sel. Areas Commun. 17(8), 1353-1368 (2002)

8. E Fasolo, A Zanella, M Zorzi, An effective broadcast scheme for alert message propagation in vehicular ad hoc networks, in Proc. IEEE International Conf. on Commun., vol. 9. ((ICC), Istanbul, Turkey, 2006), pp. 3960-3965

9. J Sahoo, E Wu, P Sahu, M Gerla, BPAB: binary partition assisted emergency broadcast protocol for vehicular ad hoc networks, Proc. of Intl. Conference on Computer Communications and Networks, (ICCCN), San Francisco, CA, USA, (August 2009), pp. 1-6

10. S Busanelli, G Ferrari, S Panichpapiboon, Efficient broadcasting in IEEE 802.11 networks through irresponsible forwarding, in Proc. IEEE Global Telecommun. Conf, (GLOBECOM), Honolulu, HA, USA, (Nov 30-Dec 30 2009), pp. 1-6

11. AM Hanashi, A Siddique, I Awan, M Woodward, Performance evaluation of dynamic probabilistic broadcasting for flooding in mobile ad hoc networks. Simul. Model. Pract. Theory. 17(2), 364-375 (2009). doi:10.1016/j. simpat.2008.09.012

12. N Wisitpongphan, O Tonguz, J Parikh, P Mudalige, F Bai, V Sadekar, Broadcast storm mitigation techniques in vehicular ad hoc networks. IEEE Wirel. Commun. Mag.. 14(6), 84-94 (2007)

13. S Busanelli, G Ferrari, VA Giorgio, On the effects of mobility for efficient broadcast data dissemination in $12 \mathrm{~V}$ networks, in Proc. SWiM Workshop IEEE Global Telecommun. Conf., (GLOBECOM), Miami, FL, USA, (2010), pp. 38-42

14. N Wisitpongphan, F Bai, P Mudalige, V Sadekar, OK Tonguz, Routing in sparse vehicular ad hoc wireless networks. IEEE J. Sel. Areas Commun. 25(8), 1538-1556 (2007)

15. A Papoulis, Probability, Random Variables and Stochastic Processes, 3rd edn. (McGraw-Hill, New York, 2001)

16. Insitute of Electrical and Electronics Engineers, IEEE Std 802. 11TM-2007., (2007) Part 11: Wireless LAN Medium Access Control (MAC) and Physical Layer (PHY) specifications
17. IEEE Standard for Information technology-Telecommunications and information exchange between systems- Local and metropolitan area networks-Specific requirements Part 11: Wireless LAN Medium Access Control (MAC) and Physical Layer (PHY) Specifications Amendment 6: Wireless Access in Vehicular Environments. IEEE Std 802.11p-2010 (Amendment to IEEE Std 802.11-2007), 1-51 (2010)

18. P Mohan, VN Padmanabhan, R Ramjee, Nericell: rich monitoring of road and traffic conditions using mobile smartphones, in ACM Int. Conf. on Embedded Networked Sensor Systems, (SenSys), Raleigh, NC, USA, (2008), pp. 323-336

19. G Ferrari, S Busanelli, N lotti, Y Kaplan, Cross-network information dissemination in VANETs, in Proc. of IEEE Intl. Conf. on ITS Telecommunications, (ITST), Saint-Petersburg, Russia, 2011), pp. 133-139

20. S Panichpapiboon, G Ferrari, Irresponsbile forwarding, in Proc. IEEE Intl. Conf. on Intelligent Transport System Telecommunication, (ITST), Phuket, Thailand, 2008), pp. 311-316

21. T Kosch, I Kulp, M Bechler, M Strassberger, B Weyl, R Lasowski, Communication architecture for cooperative systems in Europe. IEEE Commun. Mag. 47(5), 116-125 (2009)

22. Network Simulator 2 (ns-2), (Available at) http://isi.edu/nsnam/ns/.

23. Q Chen, F Schmidt-Eisenlohr, D Jiang, M Torrent-Moreno, L Delgrossi, H Hartenstein, Overhaul of IEEE 802.11 modeling and simulation in ns-2, in Proc. of the ACM Symposium on Modeling, Analysis, and Simulation of Wireless and Mobile Systems, (MSWiM), Chania, Crete Island, Greece, (2007), pp. 159-168

24. M Fiore, J Härri, F Filali, C Bonnet, Vehicular mobility simulation for VANETs, in Proc. of SCS Annual Simulation Symposium, (ANSS), Norfolk, VA, USA 2007), pp. 301-309

25. VanetMobiSim Project, http://vanet.eurecom.fr/

26. S Busanelli, G Ferrari, VA Giorgio, I2V highway and urban vehicular networks: a comparative analysis of the impact of mobility on broadcast data dissemination. J. Comm. SI Seam. Mobil. Wirel. Netw.. 6, 87-100 (2011)

27. S Busanelli, G Ferrari, S Panichpapiboon, Cluster-based irresponsible forwarding, in The Internet of Things, 20th Tyrrhenian International Workshop on Digital Communications, Springer, ed. by D Giusto, G Morabito, A lera, L Atzori Pula, Sardinia, Italy, (Sep 2009)

28. Eureka Project 6252 X-NETAD, Http://www.eurekanetwork.org/project/-/id/ 6252

29. W Feller, in An Introduction to Probability Theory and its Applications, vol. 1. (Wiley, New York, 1968)

doi:10.1186/1687-1499-2012-10

Cite this article as: Busanelli et al.: Recursive analytical performance evaluation of broadcast protocols with silencing: application to VANETs. EURASIP Journal on Wireless Communications and Networking 2012 2012:10.

\section{Submit your manuscript to a SpringerOpen ${ }^{\circ}$ journal and benefit from:}

- Convenient online submission

- Rigorous peer review

- Immediate publication on acceptance

- Open access: articles freely available online

- High visibility within the field

- Retaining the copyright to your article

Submit your next manuscript at $>$ springeropen.com 